\title{
Package 'isa2'
}

February 21, 2023

Version 0.3.6

Title The Iterative Signature Algorithm

Author Gabor Csardi <csardi.gabor @gmail.com>

Maintainer Gabor Csardi <csardi.gabor@gmail.com>

Description The ISA is a biclustering algorithm that finds modules in an input matrix. A module or bicluster is a block of the reordered input matrix.

Imports grDevices, lattice, stats, utils

Depends methods

Suggests igraph $(>=0.5 .5)$, biclust

License CC BY-NC-SA 4.0

URL https://github.com/gaborcsardi/ISA

BugReports https://github.com/gaborcsardi/ISA/issues

NeedsCompilation yes

Repository CRAN

Date/Publication 2023-02-21 21:30:02 UTC

\section{R topics documented:}

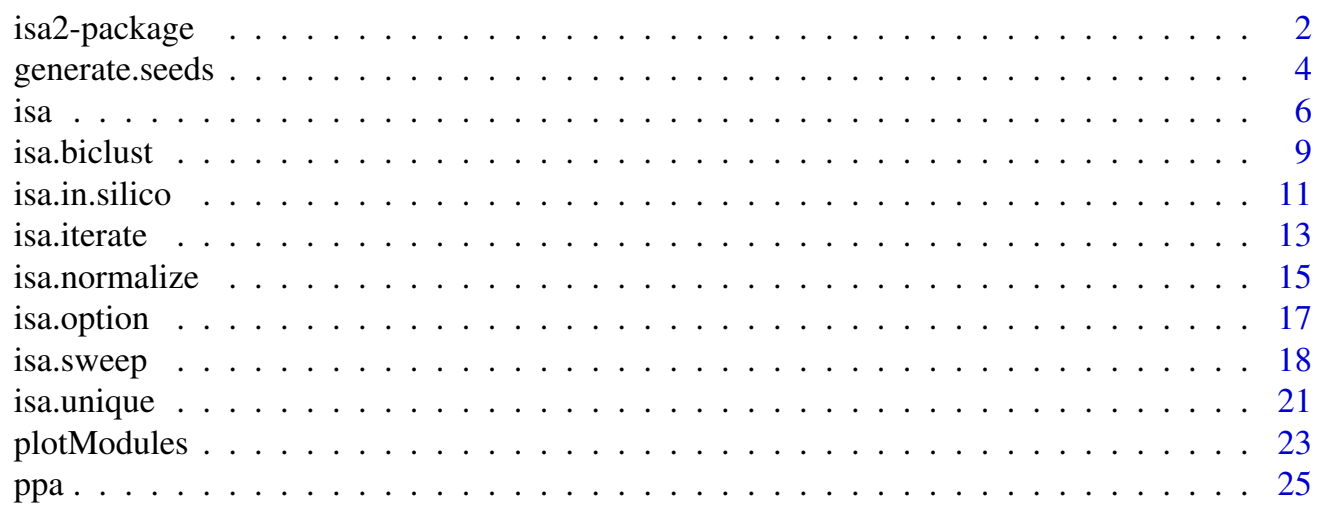


ppa.in.silico . . . . . . . . . . . . . . . . . . . . . . . . . . . . . . 29

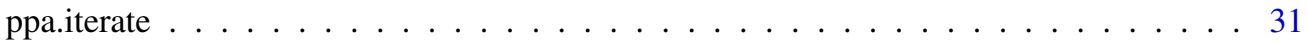

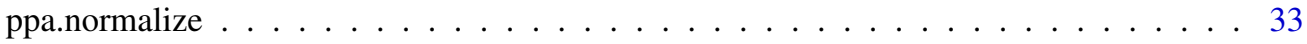

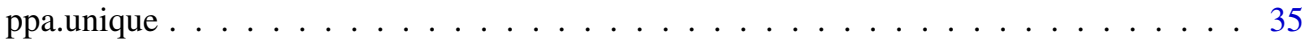

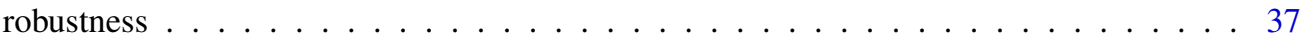

Index

isa2-package

The isa package

\section{Description}

The Iterative Signature Algorithm

\section{Introduction}

The Iterative Signature Algorithm (ISA) is a biclustering algorithm. Biclustering algorithms classify simultaneously the rows and columns of an input matrix into biclusters, or as we will call them here, modules.

\section{For the impatient}

The easiest way to run ISA is to call the is a function with your input matrix as the single argument. This does all steps of a typical ISA work flow, with the default parameters.

\section{ISA biclusters}

An ISA module is pair; a subset of the rows of the input matrix and a subset of its columns. In other words, a bicluster is a block of the reordered input matrix, where reordering means a permutation of both the rows and columns. (Another bicluster might be block of the same permuted input matrix or one after a different permutation.)

The criteria of a good bicluster is that 1 ) its rows are significantly different than the other rows, when we consider only the positions defined by the columns of the same bicluster, and (symmetrically) 2) its columns are significantly different than the other columns, when we consider only the positions defined by the rows of the same bicluster.

In other words, the rows of the bicluster are correlated, but only on the columns defined by the same bicluster; and the opposite is also true, the columns of the bicluster are correlated, but only on the rows defined by the same bicluster.

ISA biclusters are soft, two biclusters may overlap in their rows, columns or even both. It is also possible that some rows and/or columns of the input matrix are not found to be part of any ISA biclusters. Depending on the stringency parameters, it might even happen that ISA does not find any biclusters.

\section{ISA row and column scores}

ISA biclusters are not only soft, but every row and column in a given bicluster has a score, a number between minus one and one. The further this number is from zero, then stronger is the association of the given row or column to the bicluster. 


\section{How ISA works}

ISA works in an iterative way. For an $E(m \times n)$ input matrix it starts from seed vector $r_{0}$, which is typically a sparse $0 / 1$ vector of length $m$. This defines a set of rows in $E$. Then $E^{\prime}$ is multiplied by $r_{0}$ and the result is thresholded. (Please see also 'Normalization' below.)

The thresholding is an important step of the ISA, without thresholding ISA would be equivalent to a (not too effective) numerical singular value decomposition (SVD). Currently thresholding is done by calculating the mean and standard deviation of the vector and keeping only elements that are further than a given number of standard deviations from the mean. Based on the direction parameter, this means 1) keeping values that are significantly higher than the mean (direction="up"), significantly lower (direction="down") or both (direction="updown").

The thresholded vector $c_{0}$ is the (column) 'signature' of $r_{0}$. Then the (row) signature of $c_{0}$ is calculated, $E$ is multiplied by $c_{0}$ and then thresholded to get $r_{1}$.

This iteration is performed until it converges, i.e. $r_{i}$ and $r_{i-1}$ are "close", and $c_{i}$ and $c_{i-1}$ are also close. The convergence criteria, i.e. what "close" means is by default defined by high Pearson correlation.

It is very possible that the ISA finds the same modules more than once; two or more seeds might converge to the same module. The function isa. unique eliminates every module from the result of isa.iterate that is very similar (in terms of Pearson correlation) to the one that was already found before it.

\section{Parameters}

The two main parameters of ISA are the two thresholds (one for the rows and one for the columns). They basically define the stringency of the modules. If the row threshold is high, then the modules will have very similar rows. If it is mild, then modules will be bigger, with less similar rows than in the first case.

\section{Random seeding and smart seeding}

By default (i.e. if the isa function is used) the ISA is performed from random sparse starting seeds, generated by generate.seeds. This way the algorithm is completely unsupervised, but also stochastic: it might give different results for different runs.

It is possible to use non-random seeds as well, if you have some knowledge about the data or are interested in a particular subset of rows/columns, then you can feed in your seeds into the isa.iterate function directly. In this case the algorithm is deterministic, for the same seed you will always get the same results.

\section{Normalization}

On in silico data we observed that ISA has the best performance if the input matrix is normalized (see isa.normalize). The normalization produces two matrices: $E_{r}$ and $E_{c} . E_{r}$ is calculated by transposing $E$ and centering and scaling its rows (see scale). $E_{c}$ is calculated by centering and scaling the rows of $E$. $E_{r}$ is used to calculate the column signature of rows and $E_{c}$ is used to calculate the signature of the columns.

It is possible to use another normalization, then the user is requested to supply the normalized input data in a named list, including the two matrices of appropriate dimensions. 'Er' will be used for 
calculating the signature of the rows, 'Ec' the signature of the columns. If you want to use the same matrix in both steps, then supply it twice, the first one transposed.

\section{Robustness}

As ISA is an unsupervised algorithm, it may very well find some modules, even if you feed in noise as an input matrix. To avoid these spurious modules we defined a robustness measure, a single number for a modules that gives how well the rows and the columns are correlated.

It recommended that the user uses isa.filter. robust to run ISA on the scrambled input matrix with the same threshold parameters and then drop every module, which has a robustness score lower than the highest robustness score among modules found in the scrambled data.

\section{A typical ISA work flow}

Please see the manual page and the source code of isa for a typical ISA work flow. (You can obtain the source code by typing 'isa' (without the apostrophes) into your R prompt and pressing ENTER.)

\section{Author(s)}

Gabor Csardi <Gabor. Csardi@unil. ch>

\section{References}

Bergmann S, Ihmels J, Barkai N: Iterative signature algorithm for the analysis of large-scale gene expression data Phys Rev E Stat Nonlin Soft Matter Phys. 2003 Mar;67(3 Pt 1):031902. Epub 2003 Mar 11.

Ihmels J, Friedlander G, Bergmann S, Sarig O, Ziv Y, Barkai N: Revealing modular organization in the yeast transcriptional network Nat Genet. 2002 Aug;31(4):370-7. Epub 2002 Jul 22

Ihmels J, Bergmann S, Barkai N: Defining transcription modules using large-scale gene expression data Bioinformatics 2004 Sep 1;20(13):1993-2003. Epub 2004 Mar 25.

\section{See Also}

The vignette in the package and isa for running ISA.

\section{Description}

Generate random input seeds for the ISA.

\section{Usage}

generate. seeds (length, count $=100$, method $=c(" u n i ")$, sparsity=2) 


\section{Arguments}

length

The length of the seeds, should be the number of rows in your input data for row seeds and the number of columns for column seeds.

count The number of seeds to gnerate.

method The method for generating the seeds. Currently only "uni" is supported, that picks the 1 elements in each seed uniformly randomly.

sparsity A numeric scalar, an integer number giving the number of non-zero values in each seed vector. It will be recycled to have the same length as the number of seeds.

\section{Details}

This function can generate a 0/1 matrix whose columns are the seeds of the ISA. The result can be use as the row. seeds (or col.seeds) argument of the isa. iterate function.

\section{Value}

A numeric matrix with $0 / 1$ values.

\section{Author(s)}

Gabor Csardi <Gabor. Csardi@unil.ch>

\section{References}

Bergmann S, Ihmels J, Barkai N: Iterative signature algorithm for the analysis of large-scale gene expression data Phys Rev E Stat Nonlin Soft Matter Phys. 2003 Mar;67(3 Pt 1):031902. Epub 2003 Mar 11.

Ihmels J, Friedlander G, Bergmann S, Sarig O, Ziv Y, Barkai N: Revealing modular organization in the yeast transcriptional network Nat Genet. 2002 Aug;31(4):370-7. Epub 2002 Jul 22

Ihmels J, Bergmann S, Barkai N: Defining transcription modules using large-scale gene expression data Bioinformatics 2004 Sep 1;20(13):1993-2003. Epub 2004 Mar 25.

\section{See Also}

isa2-package for a short introduction on the Iterative Signature Algorithm. See isa for an easy way of running ISA.

\section{Examples}

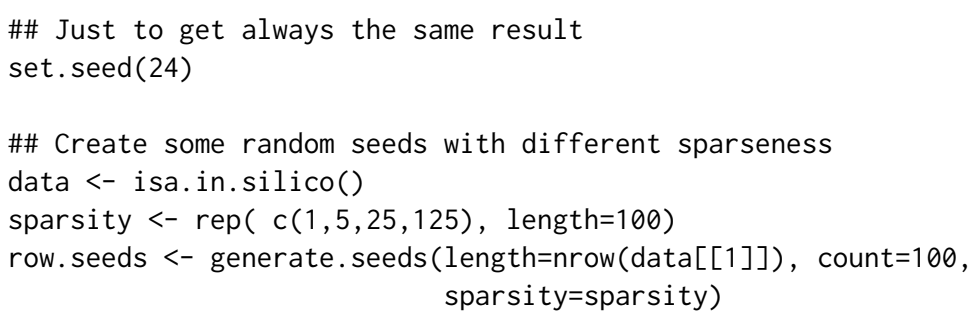




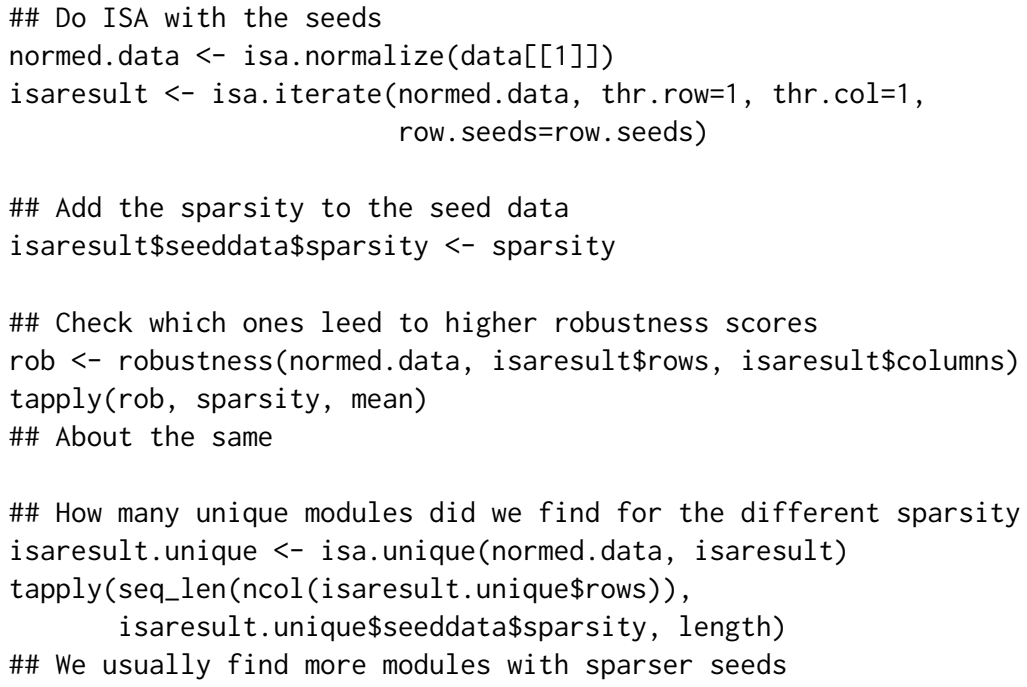

\section{isa Iterative Signature Algorithm}

\section{Description}

Run ISA with the default parameters

\section{Usage}

\#\# S4 method for signature 'matrix' isa (data, ...)

\section{Arguments}

data The input. It must be a numeric matrix. It may contain NA and/or NaN values, but then the algorithm might be a bit slower, as R matrix multiplication might be slower for these matrices, depending on your platform.

... Additional arguments, see details below.

\section{Details}

Please read the isa2-package manual page for an introduction on ISA.

This function can be called as

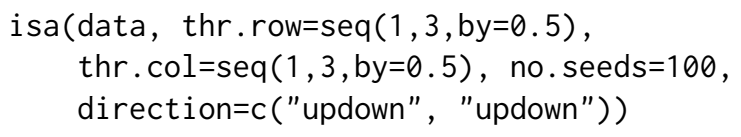


where the arguments are:

data The input. It must be a numeric matrix. It may contain NA and/or NaN values, but then the algorithm might be a bit slower, as R matrix multiplication might be slower for these matrices, depending on your platform.

thr.row Numeric vector. The row threshold parameters for which the ISA will be run. We use all possible combinations of thr. row and thr. col.

thr.col Numeric vector. The column threshold parameters for which the ISA will be run. We use all possible combinations of thr. row and thr. col.

no.seeds Integer scalar, the number of seeds to use.

direction Character vector of length two, one for the rows, one for the columns. It specifies whether we are interested in rows/columns that are higher ('up') than average, lower than average ('down'), or both ('updown').

The isa function provides an easy to use interface to the ISA. It runs all steps of a typical ISA work flow with their default parameters.

This involves:

1. Normalizing the data by calling isa.normalize.

2. Generating random input seeds via generate. seeds.

3. Running ISA with all combinations of given row and column thresholds, (by default 1, 1.5, 2, $2.5,3)$; by calling isa. iterate.

4. Merging similar modules, separately for each threshold combination, by calling isa. unique.

5. Filtering the modules separately for each threshold combination, by calling isa. filter. robust.

6. Putting all modules from the runs with different thresholds into a single object.

7. Merging similar modules, across all threshold combinations, if two modules are similar, then the larger one, the one with the milder thresholds is kept.

Please see the manual pages of these functions for the details or if you want to change their default parameters.

\section{Value}

A named list is returned with the following elements:

rows The row components in the biclusters, a numeric matrix. Every column in it corresponds to a bicluster, if an element (the score of the row) is non-zero, that means that the row is included in the bicluster, otherwise it is not. Scores are between -1 and 1 . If the scores of two rows have the same (nonzero) sign, that means that the two corresponding rows "behave" the same way. If they have opposite sign, that means that they behave the opposite way.

If the corresponding seed has not converged during the allowed number of iterations, then that column of rows contains NA values.

columns The column components of the biclusters, in the same format as the rows.

If the corresponding seed has not converged during the allowed number of iterations, then that column of columns contains NA values. 
seeddata

rundata

\section{Author(s)}

Gabor Csardi <Gabor. Csardi@unil.ch>

\section{References}

Bergmann S, Ihmels J, Barkai N: Iterative signature algorithm for the analysis of large-scale gene expression data Phys Rev E Stat Nonlin Soft Matter Phys. 2003 Mar;67(3 Pt 1):031902. Epub 2003 Mar 11.
A data frame containing information about the biclusters. There is one row for each bicluster. The data frame has the following columns:

i terations The number of iterations needed to converge to the bicluster.

oscillation The oscillation period for oscillating biclusters. It is zero for non-oscillating ones.

thr. row The row threshold that was used for find the bicluster.

thr.col The column threshold that was used for finding the bicluster.

freq The number of times the bicluster was found.

rob The robustness score of the bicluster, see robustness for details.

rob.limit The robustness limit that was used to filter the module. See isa.filter. robust for details.

A named list with information about the ISA runs. It has the following entries:

direction Character vector of length two. Specifies which side(s) of the score distribution were kept in each ISA step. See the direction argument of isa. iterate for details.

convergence Character scalar. The convergence criteria for the iteration. See the convergence argument of isa. iterate for details.

eps Numeric scalar. The threshold for convergence, if the 'eps' convergence criteria was used.

cor. limit Numeric scalar. The threshold for convergence, if the 'cor' convergence criteria was used.

corx Numeric scalar, the shift in number of iterations, to check convergence. See the convergence argument of isa. iterate for details.

maxiter Numeric scalar. The maximum number of iterations that were allowed for an input seed.

N Numeric scalar. The total number of seeds that were used for all the thresholds.

prenormalize Logical scalar. Whether the data was pre-normalized.

hasNA Logical scalar. Whether the (normalized) data had NA or NaN values.

unique Logical scalar. Whether the similar biclusters were merged by calling isa. unique.

oscillation Logical scalar. Whether the algorithm looked for oscillating modules as well.

rob.perms Numeric scalar, the number of permutations that were used to calculate the baseline robustness for filtering. See the perms argument of the isa.filter. robust function for details. 
Ihmels J, Friedlander G, Bergmann S, Sarig O, Ziv Y, Barkai N: Revealing modular organization in the yeast transcriptional network Nat Genet. 2002 Aug;31(4):370-7. Epub 2002 Jul 22

Ihmels J, Bergmann S, Barkai N: Defining transcription modules using large-scale gene expression data Bioinformatics 2004 Sep 1;20(13):1993-2003. Epub 2004 Mar 25.

\section{See Also}

isa2-package for a short introduction on the Iterative Signature Algorithm. See the functions mentioned above if you want to change the default ISA parameters.

\section{Examples}

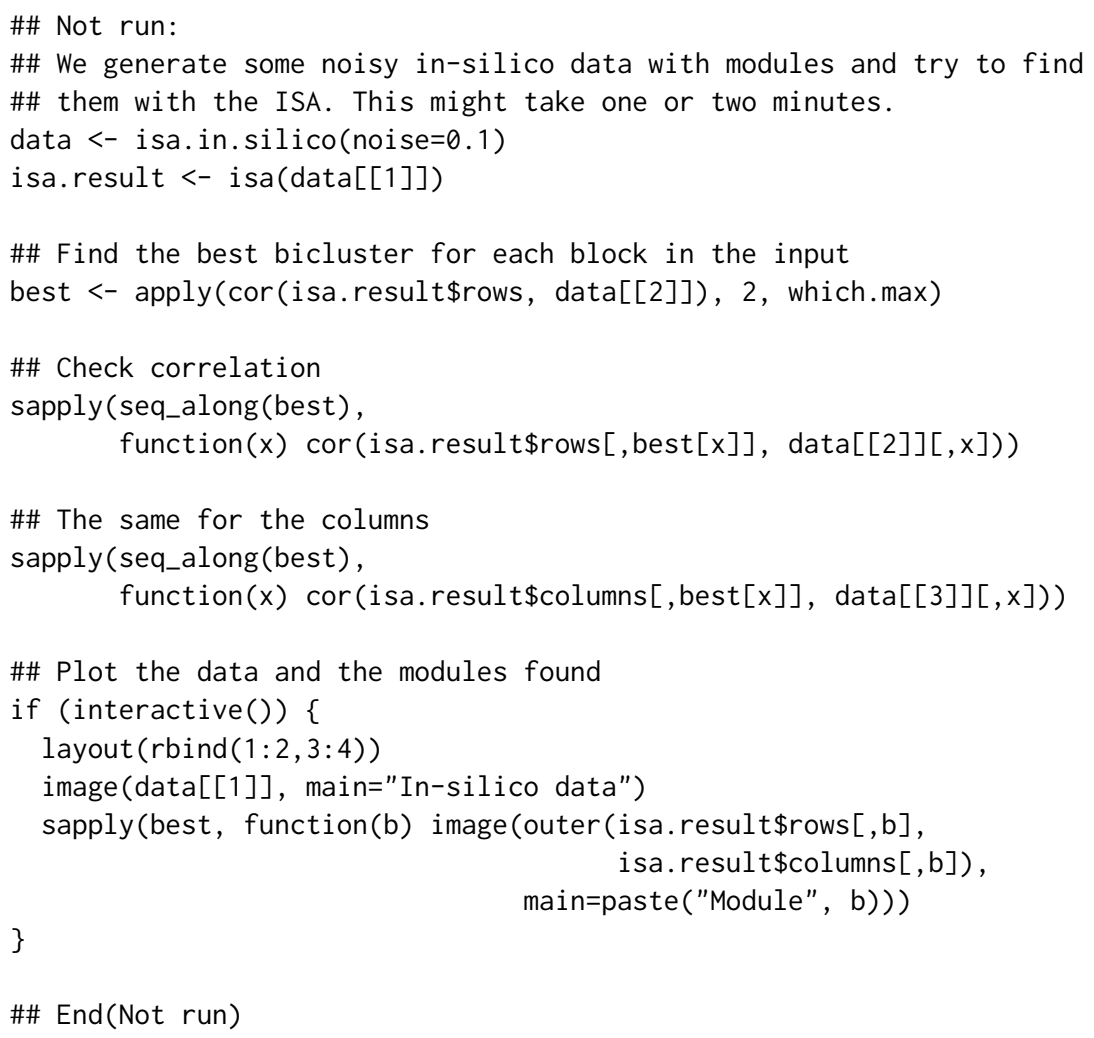

isa.biclust

Convert ISA modules to a Biclust class, as defined by the biclust package

\section{Description}

This function converts the object with ISA modules to a Biclust object, so all the functions in the biclust package can be used on it. 


\section{Usage}

isa.biclust (modules)

\section{Arguments}

modules The ISA modules, as returned by the isa or some other function.

\section{Details}

biclust is an R package that implements many biclustering algorithms in a unified framework. This function converts a set of ISA biclusters to a Biclust object, this class is used to store all biclustering results by the biclust package.

The Biclust class only supports binary biclusters, so the ISA modules are binarized during the conversion.

\section{Value}

A Biclust object.

\section{Author(s)}

Gabor Csardi <Gabor. Csardi@unil.ch>

\section{Examples}

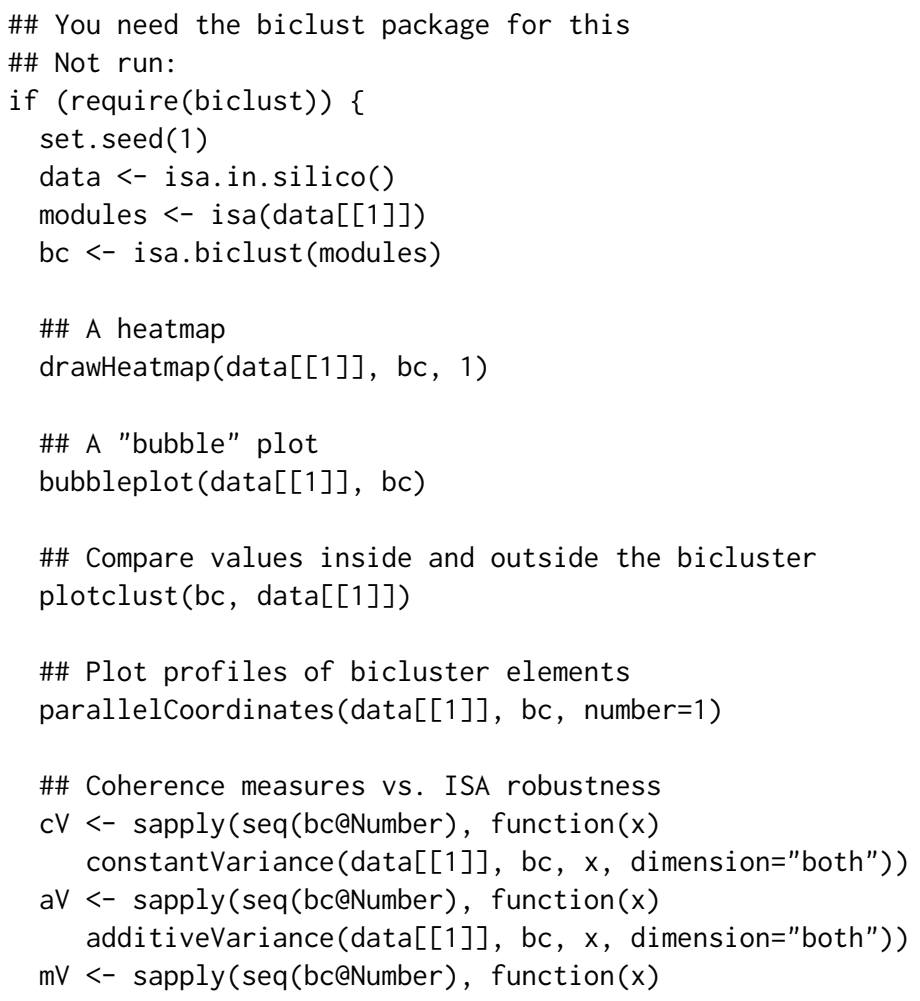




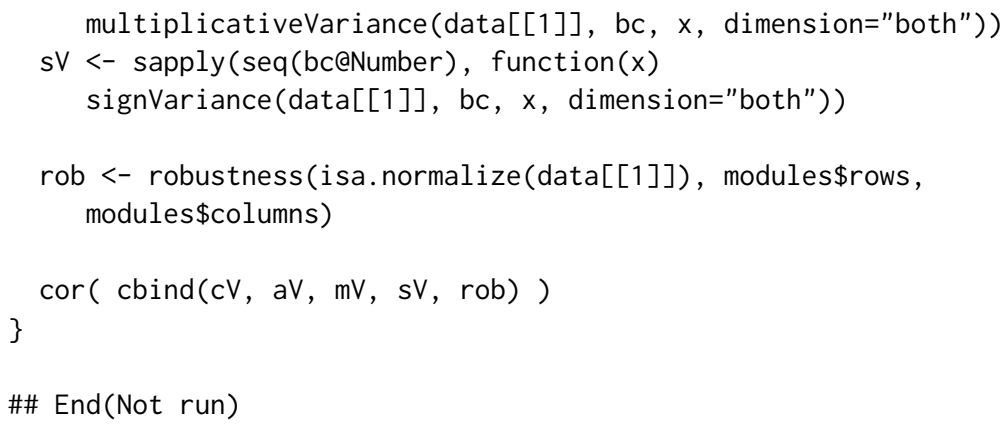

isa.in.silico

Generate in-silico input data for biclustering algorithms

\section{Description}

This function generates a test data set for ISA, containing modules of prescribed number, size, signal level, internal noise and background noise.

\section{Usage}

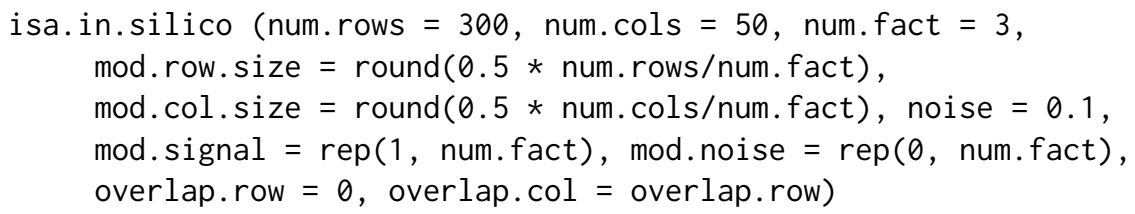

\section{Arguments}

num. rows

num.cols

num. fact

mod.row.size

mod.col.size

noise

mod.signal

mod.noise

overlap. row

overlap.col
The number of rows in the data matrix.

The number of columns in the data matrix.

The number of modules to put into the data.

The size of the modules, the number of rows per module. It can be a scalar or a vector and it is recycled.

The size of the modules, the number of columns per module. It can be a scalar or a vector and it is recycled.

The level of the background noise to be added to the data matrix. It gives the standard deviation of the normal distribution from which the noise is generated.

The signal level of the modules.

The noise levels of the different modules. Normally distributed noise with standard deviation mod. noise is added to the data. This is in addition to the background noise.

The overlap of the modules, for the rows. Zero means no overlap, one means one overlapping row, etc.

The overlap of the modules, for the columns. Zero means no overlap, one means one overlapping column, etc. 


\section{Details}

isa.in. silico creates an artificial data set to test the ISA or any other biclustering algorithm. It creates a data matrix with a checkerboard matrix. In other words, potentially overlapping blocks are planted into a noisy background matrix.

These blocks may have different signal and noise levels and they might also overlap. See the parameters above.

\section{Value}

A list with three matrices. The first matrix is the in silico data, the second contains the rows of the correct modules, the third the columns.

\section{Author(s)}

Gabor Csardi <Gabor. Csardi@unil.ch>

\section{References}

Bergmann S, Ihmels J, Barkai N: Iterative signature algorithm for the analysis of large-scale gene expression data Phys Rev E Stat Nonlin Soft Matter Phys. 2003 Mar;67(3 Pt 1):031902. Epub 2003 Mar 11.

Ihmels J, Friedlander G, Bergmann S, Sarig O, Ziv Y, Barkai N: Revealing modular organization in the yeast transcriptional network Nat Genet. 2002 Aug;31(4):370-7. Epub 2002 Jul 22

Ihmels J, Bergmann S, Barkai N: Defining transcription modules using large-scale gene expression data Bioinformatics 2004 Sep 1;20(13):1993-2003. Epub 2004 Mar 25.

\section{See Also}

isa2-package for a short introduction on the Iterative Signature Algorithm. See isa for an easy way of running ISA.

\section{Examples}

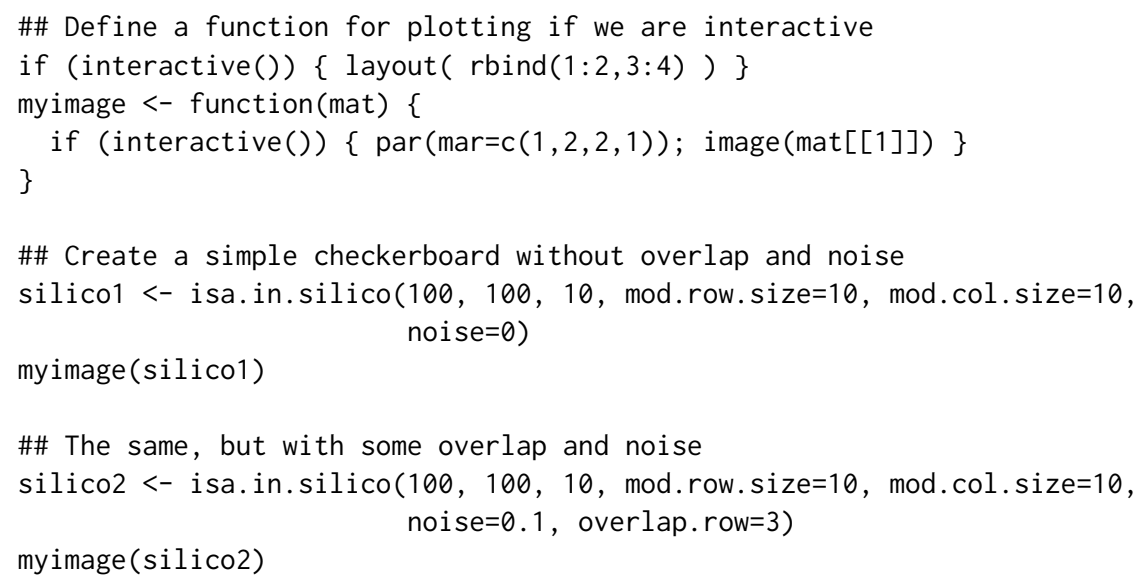




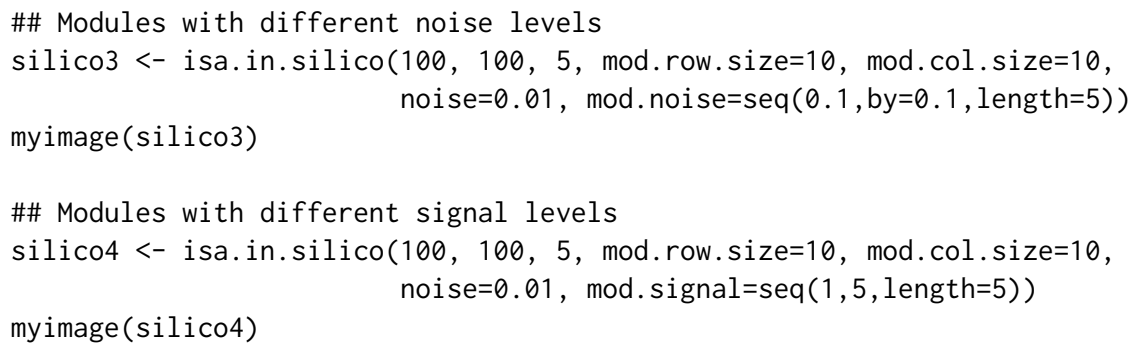

isa. iterate

The Iterative Signature Algorithm

\section{Description}

Perform ISA on the (normalized) input matrix.

\section{Usage}

\#\# S4 method for signature 'list'

isa.iterate(normed.data, ...)

\section{Arguments}

normed. data The normalized data. A list of two matrices, usually coming from isa. normalize. ... Additional arguments, see details below.

\section{Details}

isa. i terate performs the ISA iteration on the specified input seeds. It can be called as

isa.iterate(normed.data, row.seeds, col.seeds, thr. row, thr.col = thr.row, direction =c("updown", "updown"), convergence $=c($ "corx", "cor", "eps"), cor.limit $=0.99$, eps $=1 \mathrm{e}-04, \operatorname{cor} \mathrm{x}=3$, oscillation $=$ FALSE, $\operatorname{maxiter}=100$ )

where the arguments are:

normed.data The normalized data. A list of two matrices, usually coming from isa.normalize.

row.seeds The row seed vectors to start the ISA runs from. Every column is a seed vector. (If this argument and col. seeds are both present, then both of them are used.)

col.seeds The column seed vectors to start the ISA runs from, every column is a seed vector. (If this argument and row. seeds are both present, then both of them are used.) 
thr.row Numeric scalar or vector giving the threshold parameter for the rows. Higher values indicate a more stringent threshold and the result biclusters will contain less rows on average. The threshold is measured by the number of standard deviations from the mean, over the values of the row vector. If it is a vector then it must contain an entry for each seed.

thr.col Numeric scalar or vector giving the threshold parameter for the columns. The analogue of thr. row.

direction Character vector of length two, one for the rows, one for the columns. It specifies whether we are interested in rows/columns that are higher ('up') than average, lower than average ('down'), or both ('updown').

convergence Character scalar, the convergence criteria for the ISA iteration. If it is 'cor', then convergence is measured based on high Pearson correlation (see the cor. limit argument below) of the subsequent row and column vectors. If it is 'eps', then all entries of the subsequent row and column vectors must be close to each other, see the eps argument below.

'corx' is similar to 'cor', but the current row/column vectors are compared to the ones corx steps ago, instead of the ones in the previous step. See the corx argument below, that gives the size of the shift.

cor.limit The correlation limit for convergence if the 'cor' method is used.

eps Limit for convergence if the 'eps' method is used.

corx The number of iterations to use as a shift, for checking convergence with the 'corx' method.

oscillation Logical scalar, whether to look for oscillating seeds. Usually there are not too many oscillating seeds, so it is safe to leave this on FALSE.

maxiter The maximum number of iterations allowed.

Value

A named list with many components. Please see the manual page of isa for a complete description.

Author(s)

Gabor Csardi <Gabor. Csardi@unil.ch>

\section{References}

Bergmann S, Ihmels J, Barkai N: Iterative signature algorithm for the analysis of large-scale gene expression data Phys Rev E Stat Nonlin Soft Matter Phys. 2003 Mar;67(3 Pt 1):031902. Epub 2003 Mar 11.

Ihmels J, Friedlander G, Bergmann S, Sarig O, Ziv Y, Barkai N: Revealing modular organization in the yeast transcriptional network Nat Genet. 2002 Aug;31(4):370-7. Epub 2002 Jul 22

Ihmels J, Bergmann S, Barkai N: Defining transcription modules using large-scale gene expression data Bioinformatics 2004 Sep 1;20(13):1993-2003. Epub 2004 Mar 25.

\section{See Also}

isa2-package for a short introduction on the Iterative Signature Algorithm. See isa for an easy way of running ISA. 


\section{Examples}

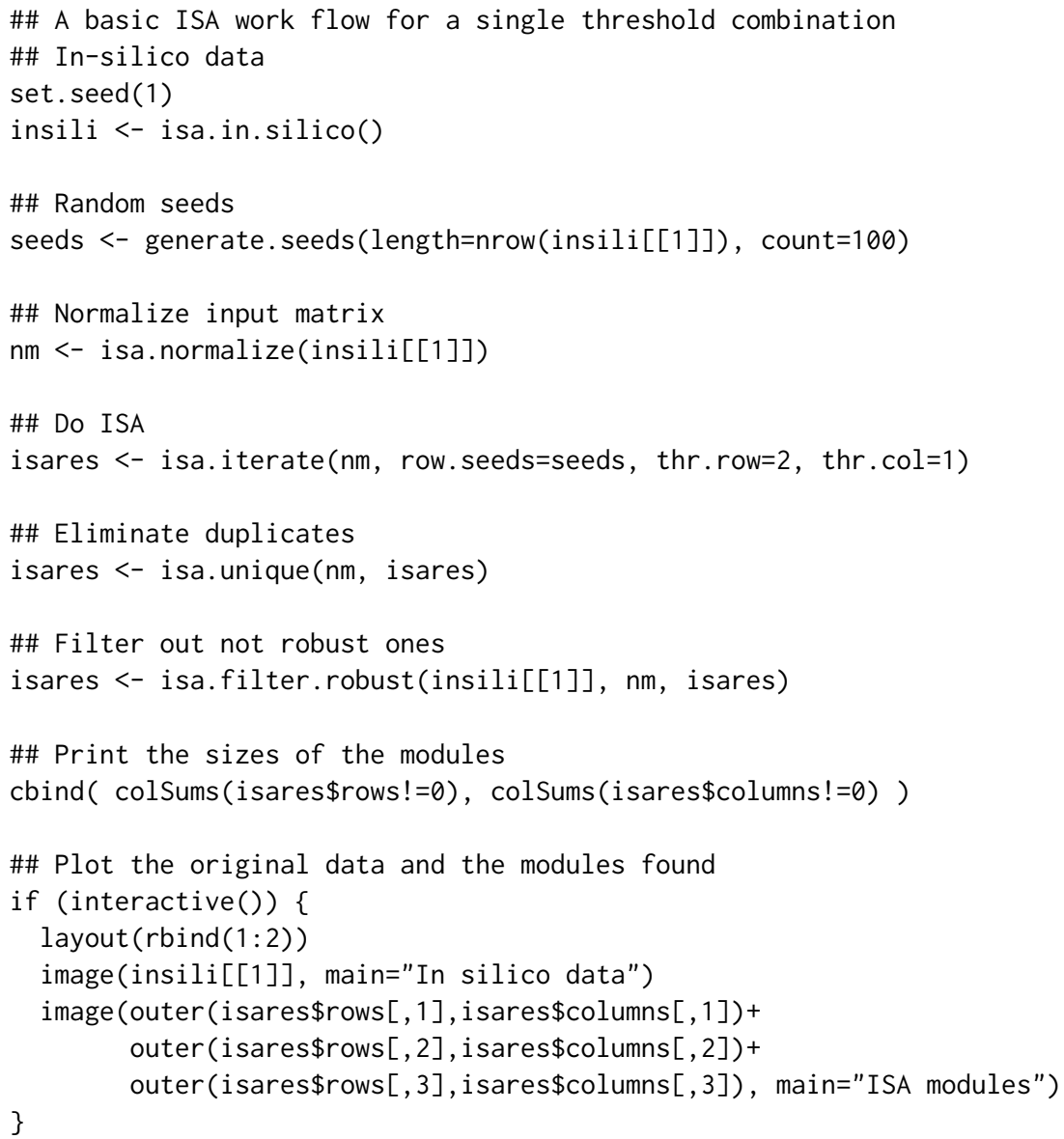

\section{Description}

Normalize a matrix and create a form that can be effectively used for ISA runs.

\section{Usage}

\#\# S4 method for signature 'matrix'

isa.normalize(data, ...)

\section{Arguments}

data
A numeric matrix, the input data. It might contains NA and/or NaN values.

Additional arguments, see details below. 


\section{Details}

This function can be called as

$$
\text { isa. normalize(data, prenormalize = FALSE) }
$$

where the arguments are:

data A numeric matrix, the input data. It might contains NA and/or NaN values.

prenormalize Logical scalar, see details below.

It was observed that the ISA works better if the input matrix is scaled and its rows have mean zero and standard deviation one.

An ISA step consists of two sub-steps, and this implies two different normalizations, in the first the rows, in the second the columns of the input matrix will be scaled.

If the prenormalize argument is set to TRUE, then row-wise scaling is calculated on the columnwise scaled matrix and not on the input matrix directly.

\section{Value}

A list of two normalized matrices, the first one is transposed.

\section{$\operatorname{Author}(\mathbf{s})$}

Gabor Csardi <Gabor. Csardi@unil.ch>

\section{References}

Bergmann S, Ihmels J, Barkai N: Iterative signature algorithm for the analysis of large-scale gene expression data Phys Rev E Stat Nonlin Soft Matter Phys. 2003 Mar;67(3 Pt 1):031902. Epub 2003 Mar 11.

Ihmels J, Friedlander G, Bergmann S, Sarig O, Ziv Y, Barkai N: Revealing modular organization in the yeast transcriptional network Nat Genet. 2002 Aug;31(4):370-7. Epub 2002 Jul 22

Ihmels J, Bergmann S, Barkai N: Defining transcription modules using large-scale gene expression data Bioinformatics 2004 Sep 1;20(13):1993-2003. Epub 2004 Mar 25.

\section{See Also}

isa2-package for a short introduction on the Iterative Signature Algorithm. See isa for an easy way of running ISA.

\section{Examples}

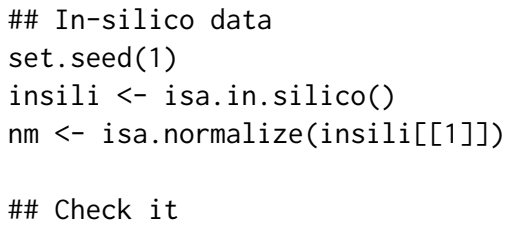




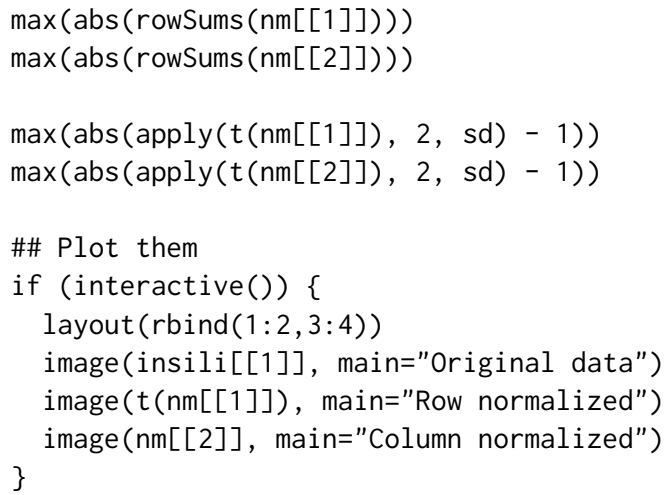

isa.option Options for the isa package

\section{Description}

This function can be used to set various options that affect many functions in the isa package.

\section{Usage}

isa.option (...)

\section{Arguments}

... A single option query, or option assignments, these must be named, too. See details below.

\section{Details}

The isa. option function can be used in three forms. First, calling it without any arguments returns a named list of the current values of all isa options.

Second, calling it with a character scalar as the single argument, it returns the value of the specified option.

Third, calling it with a named argument (or more named arguments) set the specified options to the given values.

Here is a list of all the currently supported options:

verbose Logical scalar. Whether to report what the isa functions are currently doing. Defaults to FALSE.

status. function A function object, it serves as a callback for printing status messages. 


\section{Value}

In the first form, isa.option returns a named list with the current values of all options.

In the second form, it returns the value of the specified option.

In the third form, it returns a named list with the current values of all options, invisibly.

\section{Author(s)}

Gabor Csardi <Gabor. Csardi@unil.ch>

\section{Examples}

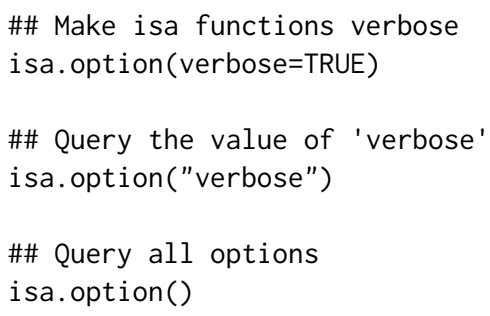

\section{Description}

Relate the biclusters found in many ISA runs on the same input data.

\section{Usage}

\#\# S4 method for signature 'matrix'

isa.sweep (data, ...)

\#\# S4 method for signature 'list'

sweep.graph (sweep.result, ...)

\section{Arguments}

data

$\cdots$

sweep. result
The input matrix.

Additional arguments, see details below. sweep.graph has no additional arguments currently.

An ISA result with hierarchy information in the seed data, typically calculated by the isa. sweep function. 


\section{Details}

isa. sweep can be called as

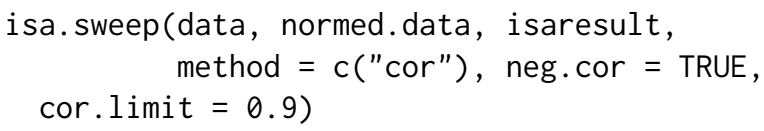

where the arguments are:

data The input matrix.

normed.data The normalized input matrix, usually the output of the isa.normalize function.

isaresult An object containing the biclusters, the result of isa or isa. iterate.

method Character scalar giving the method to determine which seed converged which bicluster. Right now only 'cor' is supported, this is based on Pearson correlation.

neg.cor Logical scalar, whether to consider negative correlation as convergence.

cor.limit Numeric scalar giving the minimum correlation for convergence.

Many ISA runs with different thresholds typically create a bunch of biclusters and it is useful to visualize how these are related.

From a set of biclusters for which of the thr. row and thr. col parameters was the same, but the other was not, isa. sweep creates a hierarchy of modules.

The hierarchy is a directed graph of modules in which every node has an out degree at most one. An edge pointing from module $m$ to module $n$ means that module $n$ is "part of" module $m$; in the sense that an ISA iteration started from module $n$ converges to module $m$ at the (milder) thresholds of module $m$.

The information about the module relationships is stored in a column of the seed data.

sweep.graph takes the output of isa. sweep and creates a graph object of it. For this the 'igraph' package is required to be installed on the system.

\section{Value}

isa.sweep returns a named list with the same components as in the input (isaresult), but the 'father' and the 'level' columns are added to the 'seeddata' member. father contains the edges of the sweep graph: if bicluster $m$ is the father of bicluster $n$ that means that bicluster $n$ converges to bicluster $m$ at the same threshold parameters that were used to find biclusters $m$.

level is a simple numbering of the different thresholds for which the sweep tree was built. I.e. the most strict threshold is level one, the second most is level two, etc.

sweep.graph returns and igraph graph with a lot of attributes:

The layout graph attribute contains a two-column matrix with the coordinates for an optimal tree-like layout to plot the graph.

The width and height graph attributes contain the optimal width and height of the plot, in inches. 
4

5

6
The id vertex attribute contains the id of the module, these correspond to the indices in the result matrix.

The rows and cols vertex attributes contain the number of rows and columns in the module.

The shape, size, size2, label vertex attributes and the arrow. size edge attribute contain various graphical parameters.

\section{Author(s)}

Gabor Csardi <Gabor. Csardi@unil.ch>

\section{References}

Bergmann S, Ihmels J, Barkai N: Iterative signature algorithm for the analysis of large-scale gene expression data Phys Rev E Stat Nonlin Soft Matter Phys. 2003 Mar;67(3 Pt 1):031902. Epub 2003 Mar 11.

Ihmels J, Friedlander G, Bergmann S, Sarig O, Ziv Y, Barkai N: Revealing modular organization in the yeast transcriptional network Nat Genet. 2002 Aug;31(4):370-7. Epub 2002 Jul 22

Ihmels J, Bergmann S, Barkai N: Defining transcription modules using large-scale gene expression data Bioinformatics 2004 Sep 1;20(13):1993-2003. Epub 2004 Mar 25.

\section{See Also}

isa2-package for a short introduction on the Iterative Signature Algorithm. See isa for an easy way of running ISA.

\section{Examples}

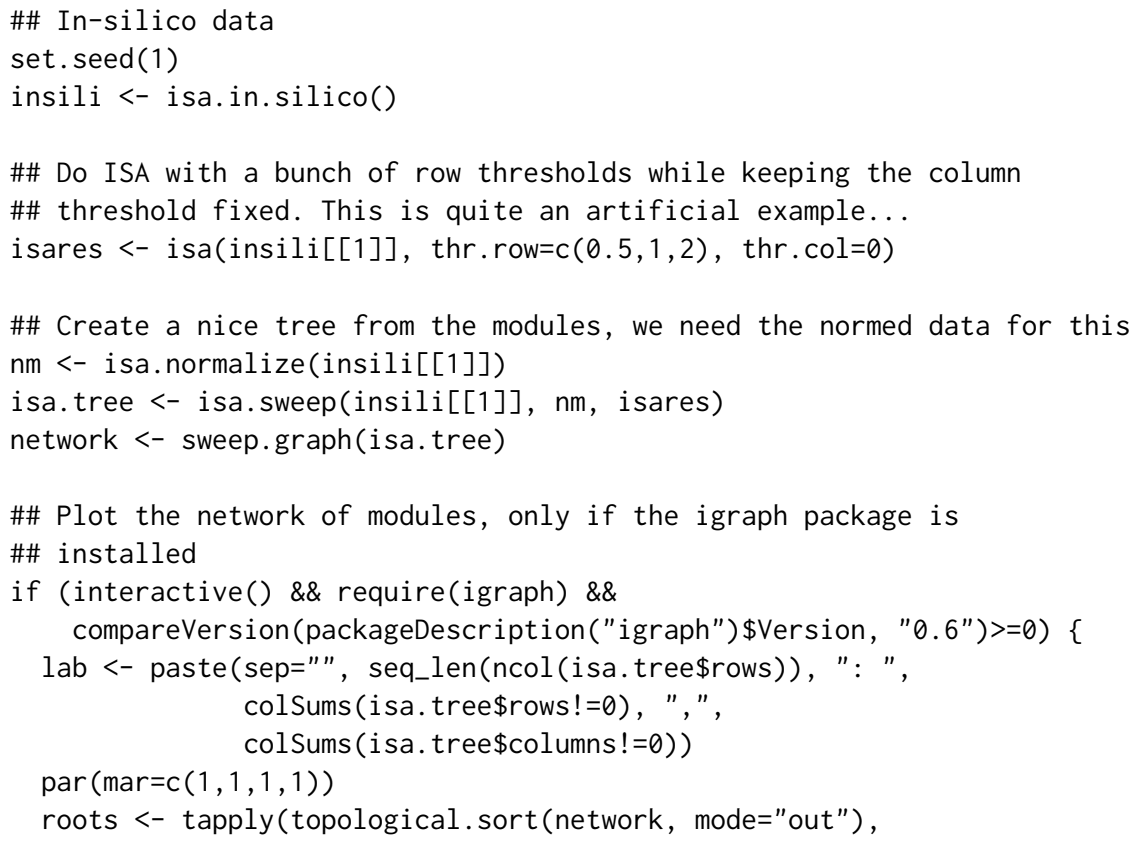




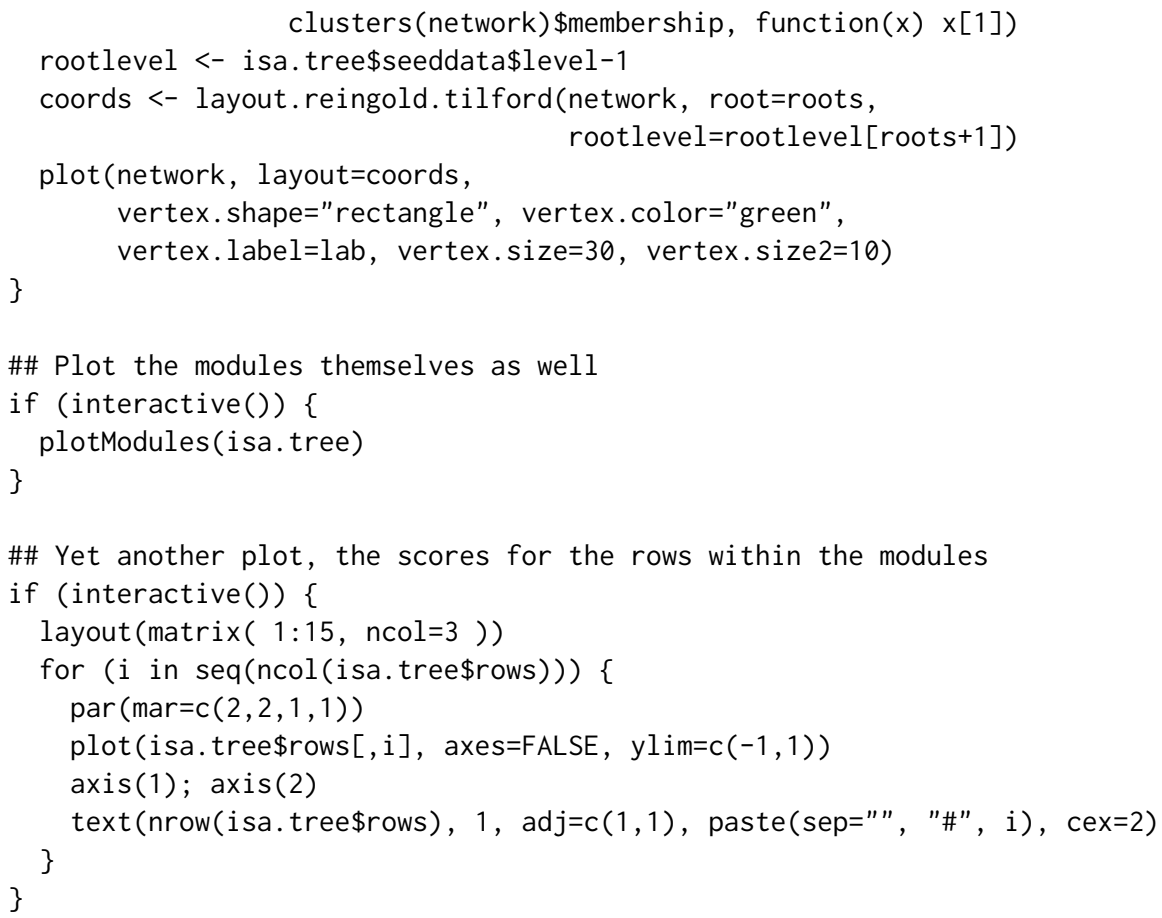

\section{Description}

From a potentially non-unique set of ISA biclusters, create a unique set by removing all biclusters that are similar to others.

\section{Usage}

\#\# S4 method for signature 'list,list'

isa.unique(normed.data, isaresult, ...)

\section{Arguments}

normed. data The normalized input data, a list of two matrices, usually the output of isa. normalize.

isaresult The result of an ISA run, a set of biclusters.

... Additional arguments, see details below. 


\section{Details}

This function can we called as

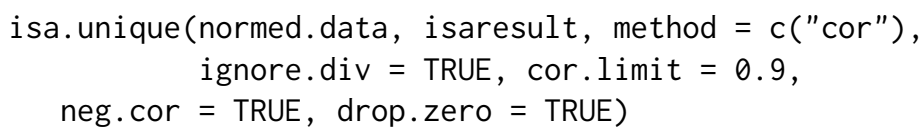

where the arguments are:

normed.data The normalized input data, a list of two matrices, usually the output of isa.normalize.

isaresult The result of an ISA run, a set of biclusters.

method Character scalar giving the method to be used to determine if two biclusters are similar. Right now only 'cor' is implemented, this keeps both biclusters if their Pearson correlation is less than cor. limit, both for their row and column scores. See also the neg. cor argument.

ignore.div Logical scalar, if TRUE, then the divergent biclusters will be removed.

cor.limit Numeric scalar, giving the correlation limit for the 'cor' method.

neg.cor Logical scalar, if TRUE, then the 'cor' method considers the absolute value of the correlation.

drop.zero Logical scalar, whether to drop biclusters that have all zero scores.

Because of the nature of the ISA algorithm, the set of biclusters created by isa.iterate is not unique; many input seeds may converge to the same biclusters, even if the input seeds are not random.

isa. unique filters a set of biclusters and removed the ones that are very similar to ones that were already found for another seed.

\section{Value}

A named list, the filtered isaresult. See the return value of isa. iterate for the details.

Author(s)

Gabor Csardi <Gabor. Csardi@unil.ch>

\section{References}

Bergmann S, Ihmels J, Barkai N: Iterative signature algorithm for the analysis of large-scale gene expression data Phys Rev E Stat Nonlin Soft Matter Phys. 2003 Mar;67(3 Pt 1):031902. Epub 2003 Mar 11.

Ihmels J, Friedlander G, Bergmann S, Sarig O, Ziv Y, Barkai N: Revealing modular organization in the yeast transcriptional network Nat Genet. 2002 Aug;31(4):370-7. Epub 2002 Jul 22

Ihmels J, Bergmann S, Barkai N: Defining transcription modules using large-scale gene expression data Bioinformatics 2004 Sep 1;20(13):1993-2003. Epub 2004 Mar 25. 


\section{See Also}

isa2-package for a short introduction on the Iterative Signature Algorithm. See isa for an easy way of running ISA.

\section{Examples}

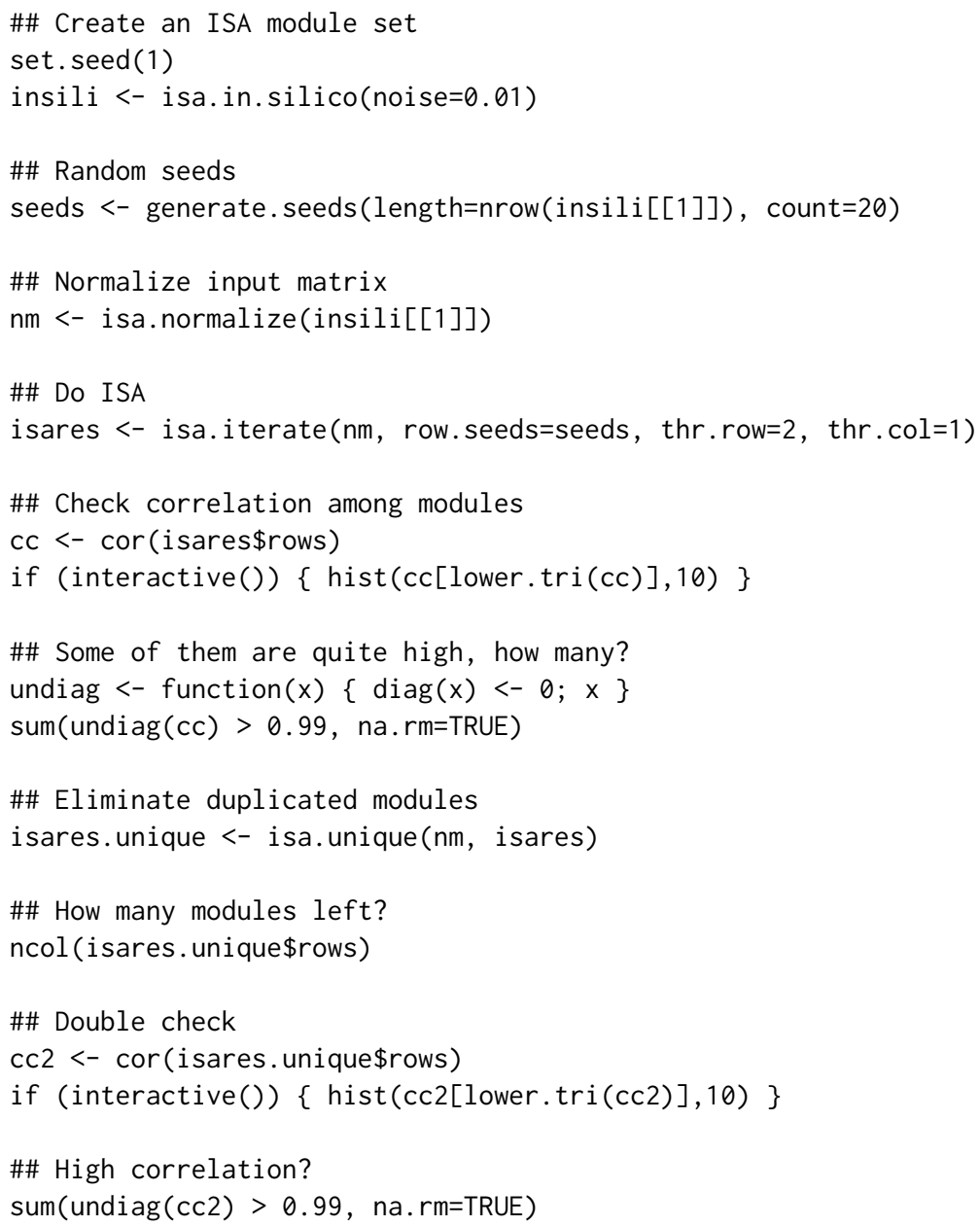

plotModules Image plots of biclusters

\section{Description}

Make several image plots, one for each bicluster, and optionally one for the original data as well. 


\section{Usage}

images (matrices, names=NULL, ...)

\#\# S4 method for signature 'list'

plotModules (modules, ...)

\section{Arguments}

matrices A list of matrices to plot. Please note that this argument is always interpreted as a list, even if want to plot a single matrix, put it into a list.

names Character vector, the labels to show above the image plots. If you give the data argument to plotModules, then the first label corresponds to that.

$\cdots$

Additional arguments, for images these are passed to levelplot, for plotModules see the details below.

modules The object with the ISA modules, as returned by the isa function or other such functions.

\section{Details}

images creates image plots for a series of matrices, using levelplot from the lattice package.

plotModules calls images from the to create image plots for a set of modules. It can be called as

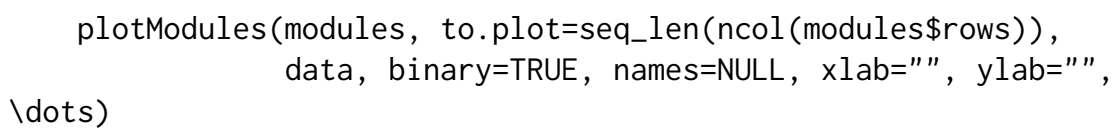

where the arguments are:

modules The object with the ISA modules, as returned by the isa function or other such functions.

to.plot Numeric vector, the modules to plot, the numbers correspond to the columns in modules $\$$ rows and modules $\$$ columns. By default all modules will be plot.

data An optional data matrix to plot. Most often this is the original data. If given, its dimension must much the dimensions in the modules object. If given, then this matrix is plotted first, before the modules.

binary Logical scalar, whether to binarize the biclusters before plotting or use the actual ISA scores. By default the biclusters are binarized.

names Character vector, the labels to show above the image plots. If you give the data argument to plotModules, then the first label corresponds to that.

xlab Character scalar, the label to put on the horizontal axis.

ylab Character scalar, the label to put on the vertical axis.

... Further arguments are passed to levelplot.

Note, that if you want to export these plots to a file, then a bitmap-based format might be more appropriate. For larger matrices vector formats tend to generate huge file because of the many dots. 
Value

Since these function use the lattice package, they return an object of class trellis. You will need to print this object to create the actual plots.

\section{$\operatorname{Author}(\mathbf{s})$}

Gabor Csardi <Gabor. Csardi@unil.ch>

\section{See Also}

image and the other version: image from the Matrix package, for alternatives to create image plots.

\section{Examples}

\#\# The following should plot the input matrix and the four modules \#\# found by ISA

set.seed(1) \# to get same plot every time

data <- isa.in.silico(100, 100, num.fact=4)

modules <- isa(data[[1]], thr. row $=2$, thr. col=2)

plotModules(modules, data=data[[1]], binary=FALSE, names $=c($ "Input matrix",

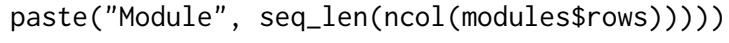

\section{Description}

Run the PPA with the default parameters

\section{Usage}

\#\# S4 method for signature 'list'

ppa(data, ...)

\section{Arguments}

data

The input, a list of two numeric matrices, with the same number of columns. They may contain NA and/or NaN values, but then the algorithm might get slower, as $\mathrm{R}$ matrix multiplication is slower sometimes slower for these matrices, depending on your platform.

Additional arguments, see details below. 


\section{Details}

Please read the isa2-package manual page for and introductino on ISA and PPA.

This function can be called as

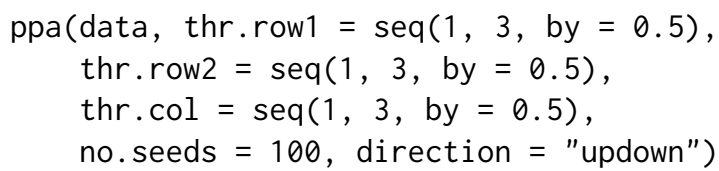

where the arguments are:

data The input, a list of two numeric matrices, with the same number of columns. They may contain NA and/or NaN values, but then the algorithm might get slower, as R matrix multiplication is slower sometimes slower for these matrices, depending on your platform.

thr.row1 Numeric scalar or vector giving the threshold parameter for the rows of the first matrix. Higher values indicate a more stringent threshold and the result comodules will contain less rows for the first matrix on average. The threshold is measured by the number of standard deviations from the mean, over the values of the first row vector. If it is a vector then it must contain an entry for each seed.

thr.row2 Numeric scalar or vector, the threshold parameter(s) for the rows of the second matrix. See thr. row1 for details.

thr.col Numeric scalar or vector giving the threshold parameter for the columns of both matrices. The analogue of thr. row1.

no.seeds Integer scalar, the number of random seeds to use.

direction Character vector of length four, one for each matrix multiplication performed during a PPA iteration. It specifies whether we are interested in rows/columns that are higher ('up') than average, lower than average ('down'), or both ('updown'). The first and the second entry both corresponds to the common column dimension of the two matrices, so they should be equal, otherwise a warning is given.

The ppa function provides and easy interface to the PPA. It runs all sptes of a typical PPA work flow, with their default paramers.

This involves:

1. Normalizing the input matrices by calling ppa. normalize.

2. Generating random input seeds via generate. seeds.

3. Running the PPA with all combinations of the given row1, row 2 and column thresholds (by default 1, 1.5, 2, 2.5, 3); by calling ppa. iterate.

4. Merging similar co-modules, separately for each threshold combination, by calling ppa. unique.

5. Filtering the co-modules separately for each threshold combination, by calling ppa. filter. robust.

6. Putting all co-modules from the run with different thresholds, into a single object.

7. Merging similar co-modules, again, but now across all threshold combinations. If two comodules are similar, then the larger one, the one with milder thresholds is kept.

Please see the manual pages of these functions for the details. 
Value

A named list is returned with the following elements:

rows1 The first components of the co-modules, corresponding to the rows of the first input matrix. Every column corresponds to a co-module, if an element (the score of the row) is non-zero, that means that that component is included in the comodule, otherwise it is not. Scores are between -1 and 1. If two scores have the same non-zero sign, then the corresponding first matrix rows are collelated. If they have an opposite sign, then they are anti-correlated.

If an input seed did not converge within the allowed number of iterations, then that column of rows 1 contains NA values. The ppa function does not produce such columns, because it always drops the non-convergent seeds via a call to ppa.unique. The result of the ppa.iterate function might contain such columns, though.

rows2

columns

seeddata

rundata
This is the same as rows 1 , but for the second input matrix.

The same as rows 1 and rows2, but for the columns of both input matrices.

A data frame containing information about the co-modules. There is one row for each co-module. The data frame has the following columns:

iterations The number of iterations needed for convergence.

oscillation The oscillation cycle of this is oscillating co-module. Zero otherwise.

thr. row1 The threshold used for the rows of the first matrix.

thr. row2 The threshold used for the rows of the second matrix.

thr.col The threshold used for the common column dimension.

freq Numeric scalar, the number of times the same (or a very similar) comodule was found.

rob The robustness score of the module.

rob. limit The robustness limit that was used to filter the module. See ppa.filter.robust for details.

A named list with information about the PPA run. It has the following entries:

direction Character vector of length four, the direction argument of the ppa.iterate call.

convergence Character scalar, the convergence criteria that was used, see the ppa.iterate function for details.

cor. limit Numeric scalar, the correlation threshold, that was used if the convergence criteria was 'cor'.

maxiter The maximum number of PPA iterations.

$\mathrm{N}$ The total number of input seeds that were used to find the co-modules.

prenormalize Logical scalar, whether the input matrices were pre-normalized, see ppa.normalize for details.

hasNA Logical vector of length two. Whether the two input matrices contained any NA or NaN values.

unique Logical scalar, whether the co-modules are unique, i.e. whether ppa. unique was called. 
oscillation Logical scalar, whether the ppa. iterate run looked for oscillating modules.

rob.perms The number of data permutations that was performed during the robustness filtering, see ppa. filter. robust for details.

\section{Author(s)}

Gabor Csardi <Gabor. Csardi@unil.ch>

\section{References}

Kutalik Z, Bergmann S, Beckmann, J: A modular approach for integrative analysis of large-scale gene-expression and drug-response data Nat Biotechnol 2008 May; 26(5) 531-9.

\section{See Also}

isa2-package for a short introduction to the ISA and the Ping-Pong algorithms. See the functions mentioned above if you want to change the default ISA parameters.

\section{Examples}

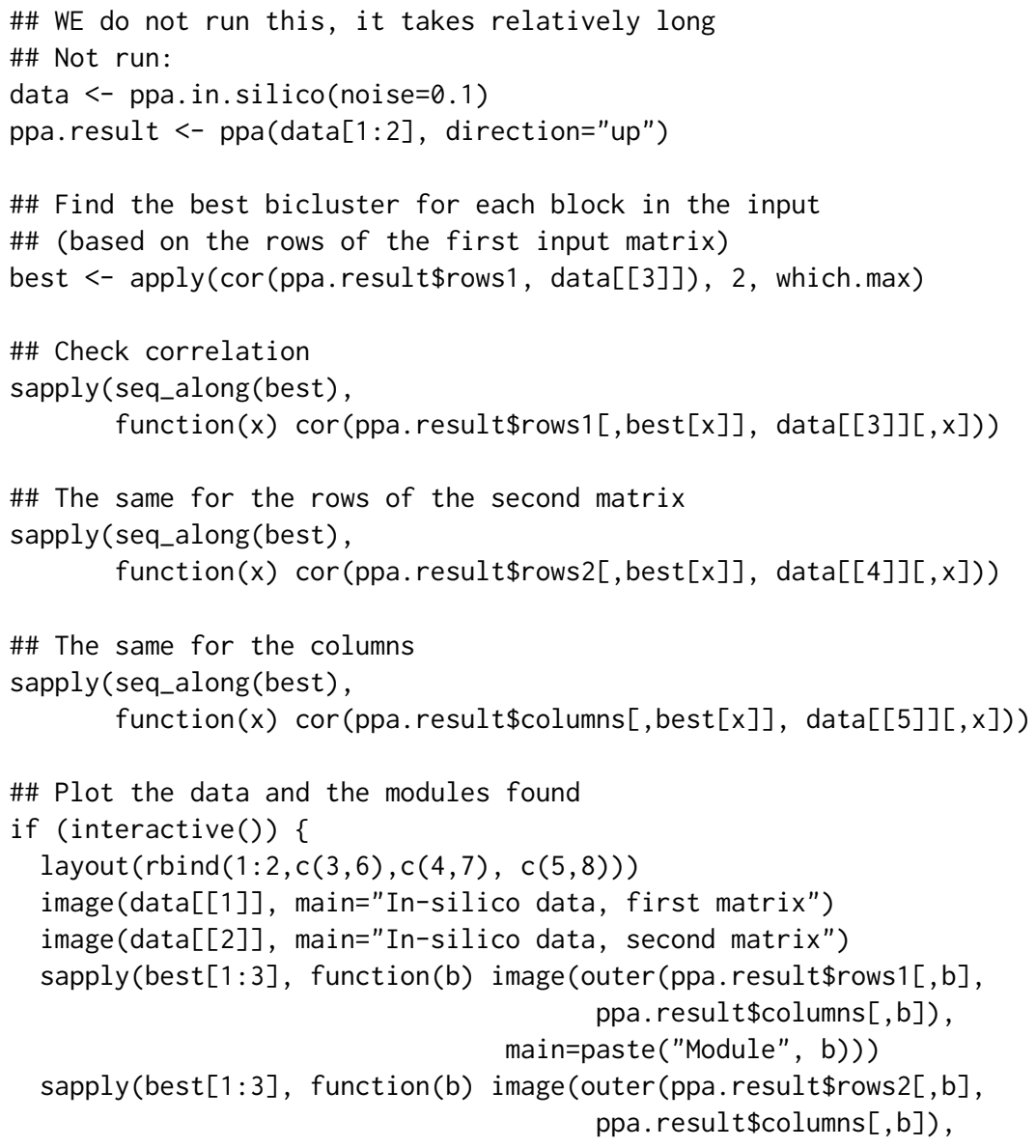


\#\# End(Not run)

ppa.in.silico

Generate in-silico input data for testing the PPA algorithm

\section{Description}

This function generates an artificial test data set for the PPA algorithm: two matrices, with common column dimension, containing co-modules of prescribed number, size, signal level, noise level and background noise.

\section{Usage}

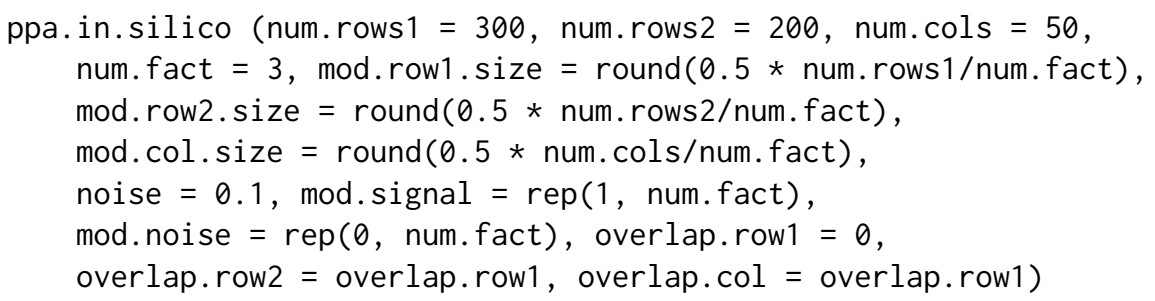

\section{Arguments}

num. rows 1

num. rows 2

num.cols

num. fact

mod. row1.size

mod.row2.size

mod.col.size

noise

mod.signal

mod.noise

overlap.row1

overlap. row2

overlap.col
The number of rows in the first data matrix.

The number of rows in the second data matrix.

The number of columns in both matrices.

The number of co-modules to put in the data.

The size of the co-modules, the number of rows from the first data matrix. It can be a scalar or a vector, and it is recycled.

The size of the co-modules, the number of rows from the second data matrix. It can be a scalar or a vector, and it is recycled.

The size of the co-modules, the number of columns (from both matrices). It can be a scalar or a vector, and it is recycled.

The level of the background noise to be added to the data matrices. It gives the standard deviation of the normal distribution from which the noise is generated. The signal level of the co-modules.

The noise levels of the different co-modules. Normally distributed noise with standard deviation mod.noise is added to the data. This is in addition to the background noise.

The overlap of the modules, for the rows of the first matrix. Zero means no overlap, one means one overlapping row, etc.

The same as overlap. row1, but for the rows of the second matrix.

The same as overlap. row1, but for the columns of both matrices. 


\section{Details}

ppa.in.silico creates an artificial data set to test the PPA (or potentially other) algorithm. It creates two data matrices with an overlapping dimension and a checkerboard-like structure. The fields of the checkerboard are the co-modules, and they may have different signal and noise levels, and they may also overlap.

\section{Value}

A list with five matrices. The first two are the two data matrices, they have the same number of columns. The last three matrices contain the correct modules, for the rows of the first matrix, the rows of the second matrix, and finally for the common column dimension.

\section{Author(s)}

Gabor Csardi <Gabor. Csardi@unil.ch>

\section{References}

Kutalik Z, Bergmann S, Beckmann, J: A modular approach for integrative analysis of large-scale gene-expression and drug-response data Nat Biotechnol 2008 May; 26(5) 531-9.

\section{See Also}

See ppa for an easy way of running the PPA

\section{Examples}

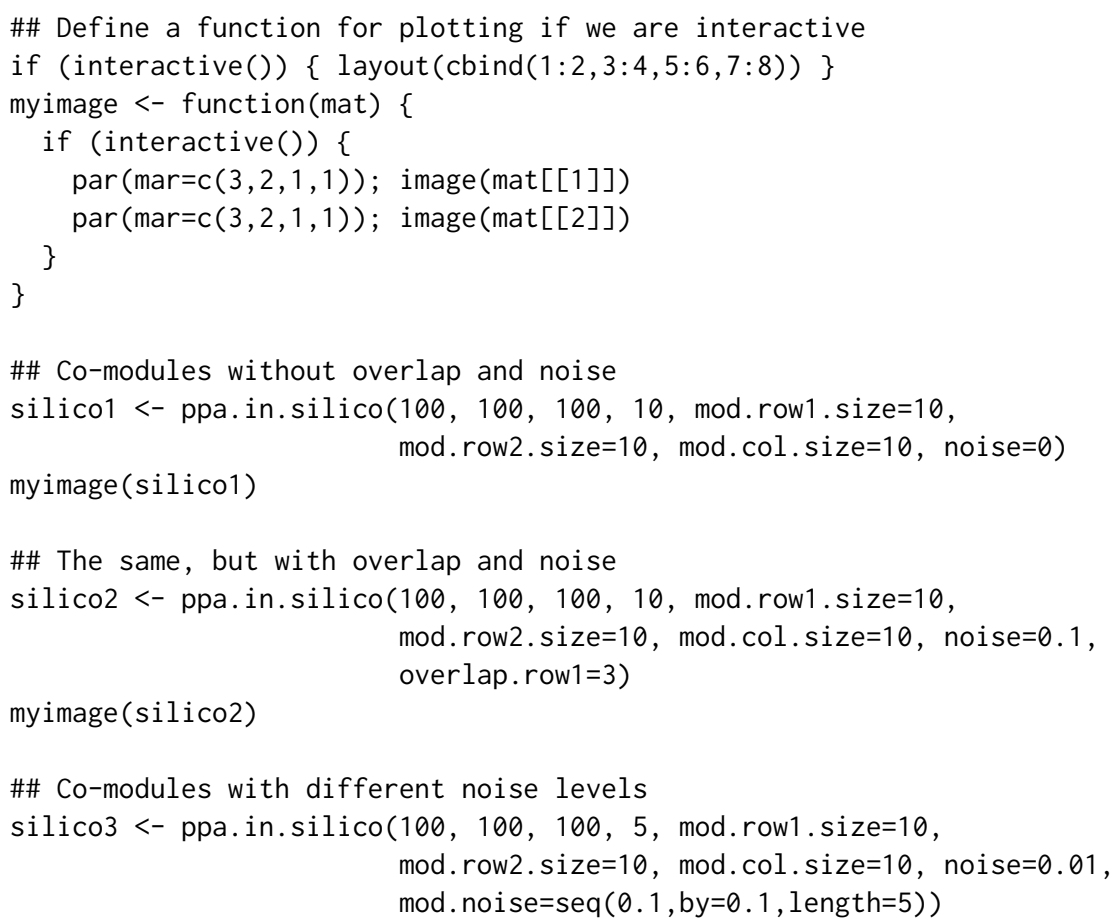


myimage(silico3)

\#\# Co-modules withe different signal levels

silico4<- ppa.in.silico(100, 100, 100, 5, mod.row1.size=10,

mod. row2. size $=10$, mod. $\operatorname{col}$. size $=10$, noise $=0.01$,

myimage(silico4)

mod. signal=seq $(1,5$, length $=5))$

ppa.iterate

The Ping-Pong Algorithm

\section{Description}

Perform PPA on two (normalized) input matrices.

Usage

\#\# S4 method for signature 'list'

ppa.iterate (normed.data, ...)

\section{Arguments}

normed.data The normalized input matrices. A list of four matrices, usually coming from the ppa.normalize function.

... Additional arguments, see details below.

\section{Details}

ppa. iterate performs the PPA iteration on the specified input matrices using given input seeds. It can be called as

ppa.iterate(normed.data, row1.seeds, col1.seeds, row2. seeds, col2. seeds, thr. row1, thr. col=thr. row1, thr. row2=thr. row1,

direction="updown",

convegence $=c("$ cor"), cor.limit=0.9,

oscillation=FALSE, maxiter=100)

where the arguments are:

normed.data The normalized data, a list of four matrices with the appropriate size. They are usually coming from the output of the ppa.normalize function.

row1.seeds The row seed vectors for the first matrix. At least one of the four possible seed vectors must be present and they will be concatenated, after doing the suitable half-iterations.

col1.seeds The column seed vectors for the first matrix. At least one of the four possible seed vectors must be present and they will be concatenated, after doing the suitable half-iterations. 
row2.seeds The row seed vectors for the second matrix. At least one of the four possible seed vectors must be present and they will be concatenated, after doing the suitable half-iterations.

col2.seeds The column seed vectors for the second matrix. At least one of the four possible seed vectors must be present and they will be concatenated, after doing the suitable half-iterations.

thr.row1 Numeric scalar or vector giving the threshold parameter for the rows of the first matrix. Higher values indicate a more stringent threshold and the result comodules will contain less rows for the first matrix on average. The threshold is measured by the number of standard deviations from the mean, over the values of the first row vector. If it is a vector then it must contain an entry for each seed.

thr.col Numeric scalar or vector giving the threshold parameter for the columns of both matrices. The analogue of thr. row1.

thr.row2 Numeric scalar or vector, the threshold parameter(s) for the rows of the second matrix. See thr. row1 for details.

direction Character vector of length four, one for each matrix multiplication performed during a PPA iteration. It specifies whether we are interested in rows/columns that are higher ('up') than average, lower than average ('down'), or both ('updown'). The first and the second entry both corresponds to the common column dimension of the two matrices, so they should be equal, otherwise a warning is given.

convergence Character scalar, the convergence criteria for the PPA iteration. If it is 'cor', then convergence is measured based on high Pearson correlation (see the cor. limit argument below) of the subsequent row and column vectors. Currently this is the only option implemented.

cor.limit The correlation limit for convergence if the 'cor' method is used.

oscillation Logical scalar, whether to look for oscillating seeds. Usually there are not too many oscillating seeds, so it is safe to leave this on FALSE.

maxiter The maximum number of iterations allowed.

Value

A named list with many components. Please see the manual page of link $\{$ isa $\}$ for a complete description.

\section{Author(s)}

Gabor Csardi <Gabor. Csardi@unil.ch>

\section{References}

Kutalik Z, Bergmann S, Beckmann, J: A modular approach for integrative analysis of large-scale gene-expression and drug-response data Nat Biotechnol 2008 May; 26(5) 531-9.

\section{See Also}

See ppa for an easy way of running the PPA 


\section{Examples}

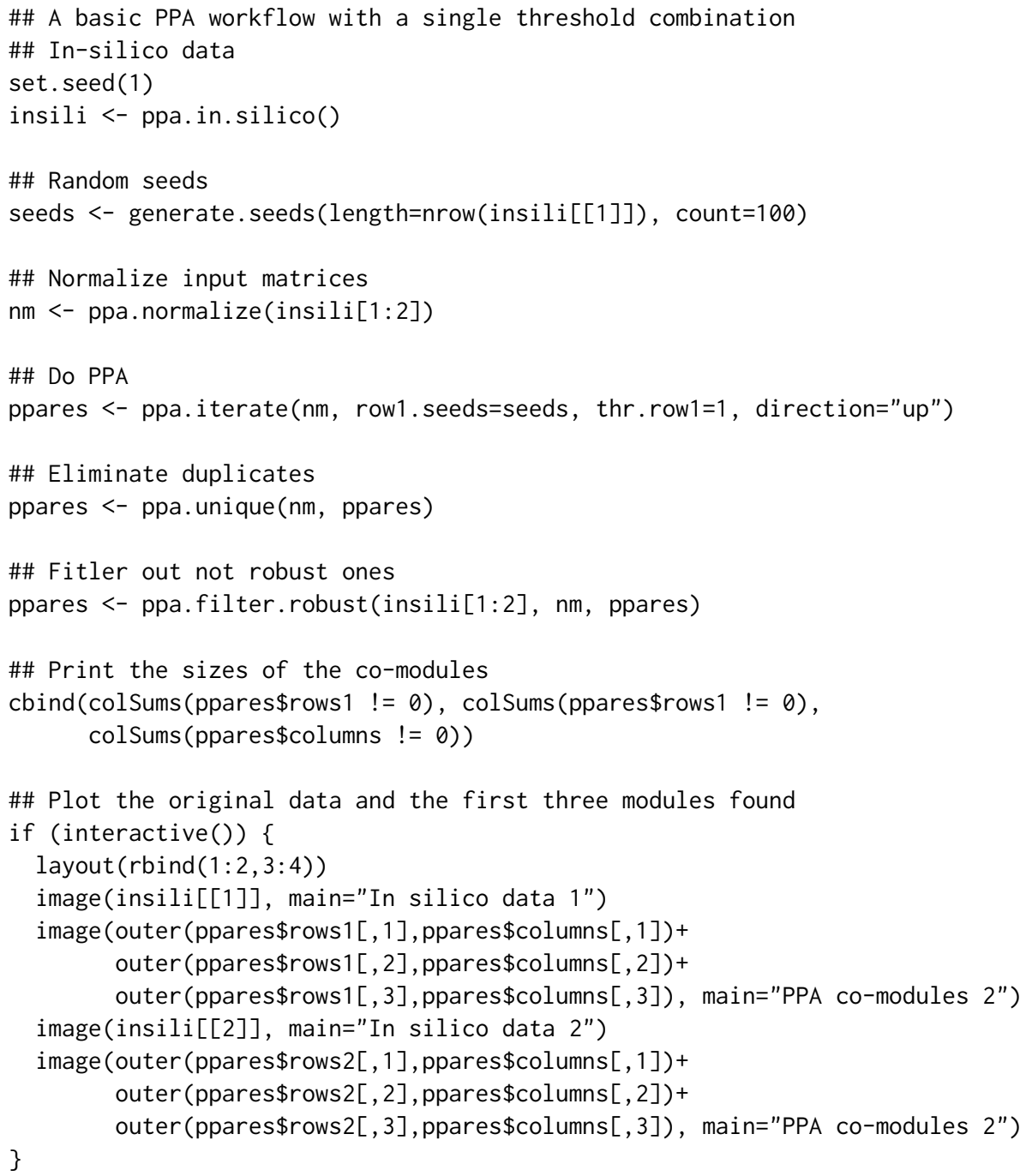

ppa.normalize Normalize input data for use with the PPA

\section{Description}

Normalize the two input matrices and store them in a form that can be used effectively to perform the Ping-Pong Algorithm

\section{Usage}

\#\# S4 method for signature 'list'

ppa.normalize(data, ...) 


\section{Arguments}

data A list of two numeric matrices, with matching number of columns. They might contain $\mathrm{Na}$ and/or $\mathrm{NaN}$ values.

... Additional arguments, see details below.

\section{Details}

This function can be called as

isa.normalize(data, prenormalize $=$ FALSE)

where the arguments are:

data A list of two numeric matrices, with matching number of columns. They might contain $\mathrm{Na}$ and/or NaN values.

prenormalize Logical scalar, see details below.

It was observed that the PPA works best if the input matrices are scaled to have mean zero and standard deviation one.

A PPA step consist of four matrix-multiplications and this requires four different matrices, each of the two input matrices scaled row-wise and column-wise.

If the prenormalized argument is set to TRUE, then row-wise scaling is calculated on the columnwise scaled matrices and not on the raw input.

\section{Value}

A list of four matrices, the first two corresponds to the first input matrix, the second two to the second matrix.

\section{Author(s)}

Gabor Csardi <Gabor. Csardi@unil.ch>

\section{References}

Kutalik Z, Bergmann S, Beckmann, J: A modular approach for integrative analysis of large-scale gene-expression and drug-response data Nat Biotechnol 2008 May; 26(5) 531-9.

\section{See Also}

See ppa for an easy way of running the PPA 


\section{Examples}

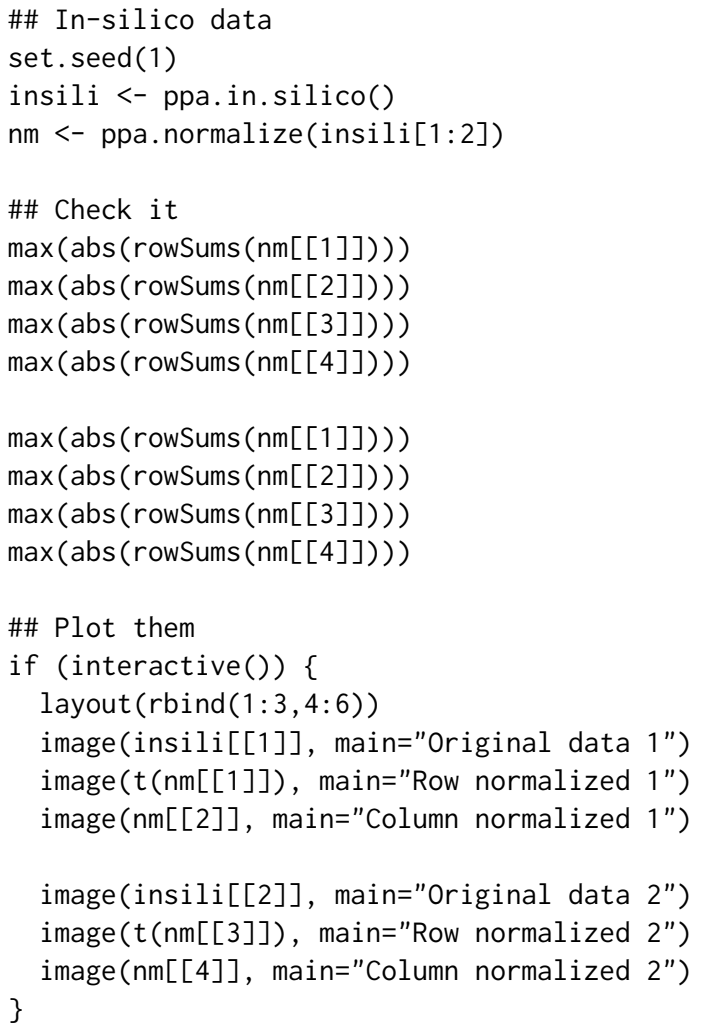

\section{Description}

From a potentially non-unique set of PPA co-modules, create a unique set by removing all comodules that are similar to others.

\section{Usage}

\#\# S4 method for signature 'list,list'

ppa.unique (normed.data, pparesult, ...)

\section{Arguments}

normed.data The normalized input data, a list of four matrices, usually the output of the ppa. normalize function.

pparesult The result of a PPA run, a set of co-modules. 


\section{Details}

This function can be called as

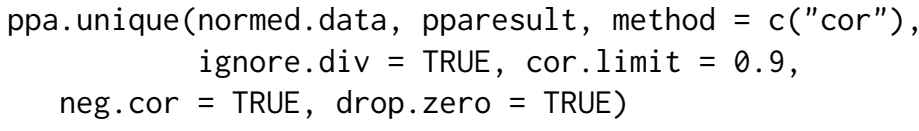

where the arguments are:

normed.data The normalized input data, a list of four matrices, usually the output of ppa. normalize.

pparesult The results of a PPA run, a set of co-modules.

method Character scalar giving the method to be used to determine if two co-modules are similar. Right now only 'cor' is implemented, this keeps both co-modules if their Pearson correlation is less than cor.limit, for their row1, row2 and column scores. See also the neg.cor argument.

ignore.div Logical scalar, if TRUE, then the divergent co-modules will be removed.

cor.limit Numeric scalar, giving the correlation limit for the 'cor' method.

neg.cor Logical scalar, if TRUE, then the 'cor' method considers the absolute value of the correlation.

drop.zero Logical scalar, whether to drop co-modules that have all zero scores.

\section{Value}

A named list, the filtered pparesult. See the return value of ppa.iterate for the details.

\section{Author(s)}

Gabor Csardi <Gabor. Csardi@unil.ch>

\section{References}

Kutalik Z, Bergmann S, Beckmann, J: A modular approach for integrative analysis of large-scale gene-expression and drug-response data Nat Biotechnol 2008 May; 26(5) 531-9.

\section{See Also}

See ppa for an easy way of running the PPA

\section{Examples}

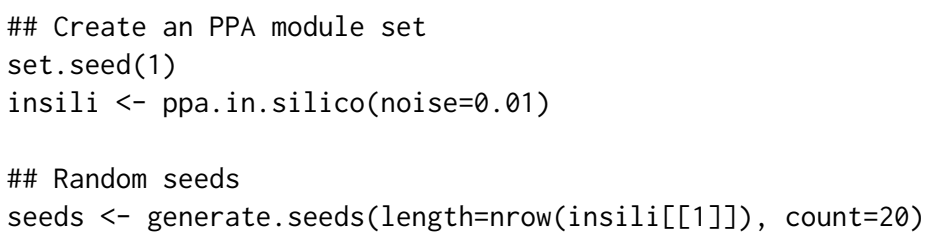




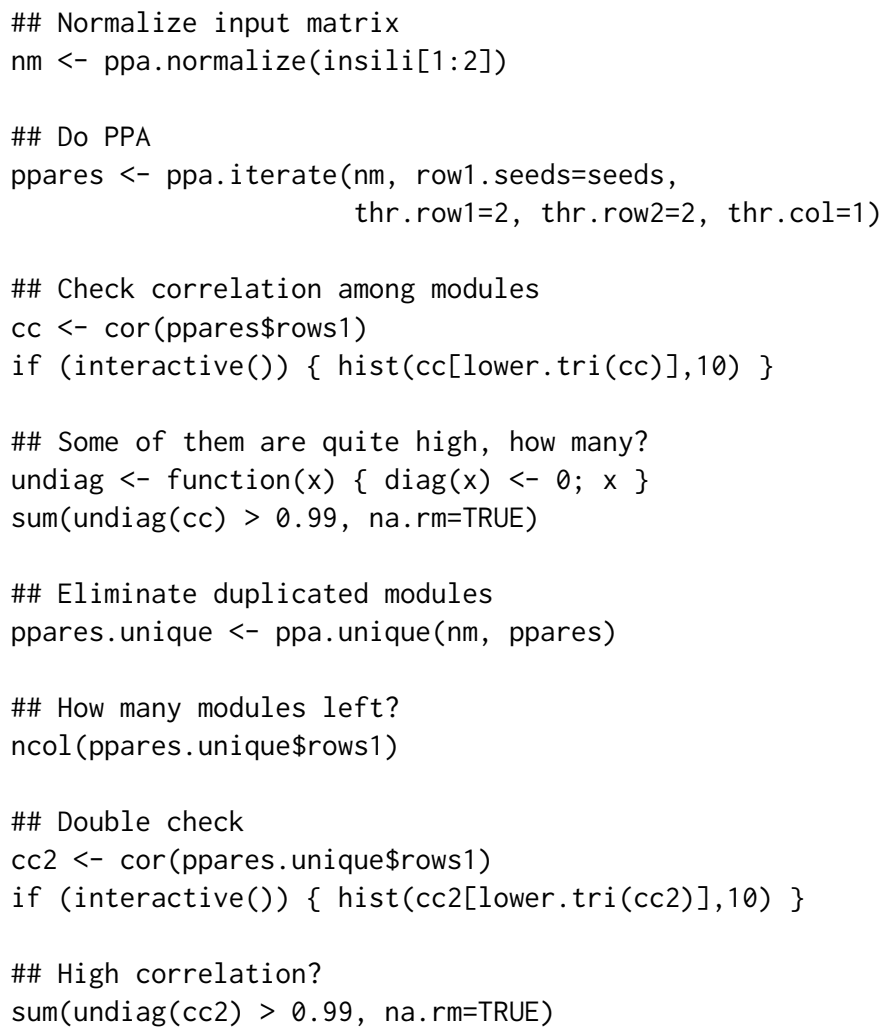

robustness Robustness of ISA biclusters and PPA co-modules

\section{Description}

Robustness of ISA biclusters and PPA co-modules. The more robust biclusters/co-modules are more significant in the sense that it is less likely to see them in random data.

\section{Usage}

\#\# S4 method for signature 'list'

robustness (normed.data, ...)

\#\# S4 method for signature 'matrix'

isa.filter. robust (data, ....)

\#\# S4 method for signature 'list'

ppa.filter.robust (data, ...)

\section{Arguments}

normed.data The normalized input data, usually calculated with isa.normalize. 
data The original, not normalized input data, a matrix for isa. filter . robust, a list of two matrices for ppa. filter. robust.

... Additional arguments, see details below.

\section{Details}

robustness can be called as

robustness(normed.data, row.scores, col.scores)

isa.filter. robust can be called as

isa.filter.robust(data, normed.data, isares, perms $=1$, row. seeds, col. seeds)

and ppa.filter. robust can be called as

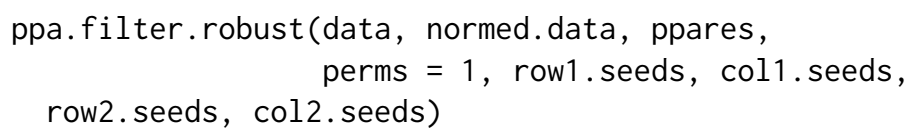

These arguments are:

normed.data The normalized input data, usually calculated with isa.normalize (for ISA) or ppa. normalize (for PPA).

row.scores The scores of the row components of the biclusters. Usually the rows member of the result list, as returned by isa, or isa. i terate or some other ISA function.

col.scores The scores of the columns of the biclusters, usually the columns member of the result list from isa.

data The original, not normalized input data.

isares The result of ISA, coming from isa or isa.iterate or any other function that return the same format.

perms The number of permutations to perform on the input data.

row.seeds Optionally the row seeds for the ISA run on the scrambled data. If this and col.seeds are both omitted the same number of random seeds are used as for isaresult.

col.seeds Optionally the column seed to use for the ISA on the scrambled input matrix. If both this and row. seeds are omitted, then the same number of random (row) seeds will be used as for isares.

ppares The result of a PPA run, the output of the ppa.iterate or the ppa.unique function (or any other function that returns the same format).

row1.seeds Optionally, the seeds based of the rows of the first input matrix, can be given here. Otherwise random seeds are used, the same number as it was used to find the original comodules. 
col1.seeds Optionally, the seeds based of the columns of the first input matrix, can be given here. Otherwise random seeds are used, the same number as it was used to find the original comodules.

row2.seeds Optionally, the seeds based of the rows of the second input matrix, can be given here. Otherwise random seeds are used, the same number as it was used to find the original comodules.

col2.seeds Optionally, the seeds based of the columns of the second input matrix, can be given here. Otherwise random seeds are used, the same number as it was used to find the original co-modules.

Even if you generate a matrix with uniform random noise in it, if you calculate ISA on it, you will get some biclusters, except maybe if you use very strict threshold parameters. These biclusters contain rows and columns that are correlated just by chance. The same is true for PPA.

To circumvent this, you can use the so-called robustness measure of the biclusters/co-modules. The robustness of a bicluster is the function of its rows, columns and the input data, and it is a real number, usually positive. It is roughly equivalent to the principal singular value of the submatrix (of the reordered input matrix) defined by the bicluster.

robustness calculates the robustness score of a set of biclusters/co-modules, usually coming from one or more ISA/PPA iterations.

isa.filter. robust provides filtering based on the robustness measure. It reshuffles the input matrix and calculates ISA on it, with the parameters that were used to find the biclusters under evaluation. It then calculates the robustness for the modules that were found in the scrambled matrix (if there is any) and removes any modules from the data set that have a lower robustness score than at least one module in the scrambled data.

You can think of isa.filter. robust as a permutation test, but the input data is shuffled only once (at least by default), because of the relatively high computational demands of the ISA.

ppa.filter. robust does essentially the same, but for PPA co-modules.

\section{Value}

robustness returns a numeric vector, the robustness score of each bicluster.

isa.filter. robust returns a named list, the filtered isares, see the return value of isa. iterate for the structure of the list.

ppa.filter. robust resturns a named list, the filtered ppares, see the return value of ppa. iterate for the structure of the list.

\section{Author(s)}

Gabor Csardi <Gabor. Csardi@unil.ch>

\section{References}

Bergmann S, Ihmels J, Barkai N: Iterative signature algorithm for the analysis of large-scale gene expression data Phys Rev E Stat Nonlin Soft Matter Phys. 2003 Mar;67(3 Pt 1):031902. Epub 2003 Mar 11.

Ihmels J, Friedlander G, Bergmann S, Sarig O, Ziv Y, Barkai N: Revealing modular organization in the yeast transcriptional network Nat Genet. 2002 Aug;31(4):370-7. Epub 2002 Jul 22 
Ihmels J, Bergmann S, Barkai N: Defining transcription modules using large-scale gene expression data Bioinformatics 2004 Sep 1;20(13):1993-2003. Epub 2004 Mar 25.

Kutalik Z, Bergmann S, Beckmann, J: A modular approach for integrative analysis of large-scale gene-expression and drug-response data Nat Biotechnol 2008 May; 26(5) 531-9.

\section{See Also}

isa2-package for a short introduction on the Iterative Signature Algorithm. See isa for an easy way of running ISA, ppa for an easy way of running the PPA.

\section{Examples}

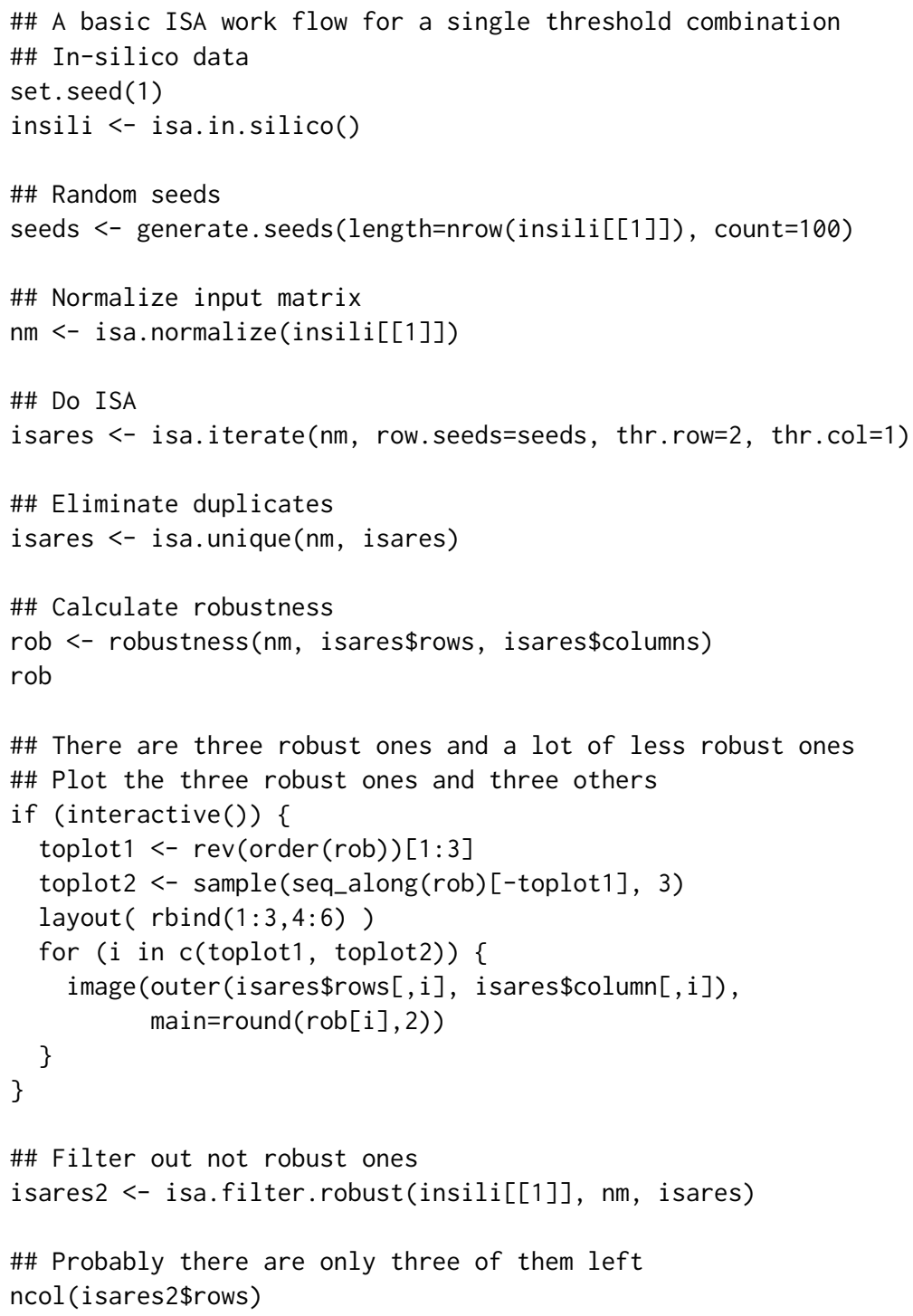




\section{Index}

$*$ Bicluster

plotModules, 23

* Iterative Signature Algorithm isa, 6

* Ping-Pong Algorithm ppa, 25

$*$ cluster

generate. seeds, 4

isa, 6

isa.in.silico, 11

isa.iterate, 13

isa. normalize, 15

isa.option, 17

isa. sweep, 18

isa. unique, 21

plotModules, 23

ppa, 25

ppa.in.silico, 29

ppa.iterate, 31

ppa. normalize, 33

ppa. unique, 35

robustness, 37

generate. seeds, $3,4,7,26$

image, 25

images (plotModules), 23

isa, $2-5,6,12,14,16,19,20,23,24,38,40$

isa, matrix-method (isa), 6

isa.biclust, 9

isa. filter. robust, $4,7,8$

isa.filter. robust (robustness), 37

isa.filter. robust, matrix-method (robustness), 37

isa.in.silico, 11

isa. i terate, $3,5,7,8,13,19,22,38,39$

isa. iterate, list-method (isa.iterate), 13

isa.normalize, $3,7,13,15,19,21,22,37,38$ isa.normalize, matrix-method

(isa.normalize), 15

isa.option, 17

isa. sweep, 18

isa. sweep, matrix-method (isa. sweep), 18

isa. unique, $3,7,8,21$

isa. unique, list, list-method (isa.unique), 21

isa2-package, 2, 5, 6, 9, 12, 14, 16, 20, 23, $26,28,40$

levelplot, 24

plotModules, 23

plotModules, list-method (plotModules), 23

ppa, 25, 30, 32, 34, 36, 40

ppa, list-method (ppa), 25

ppa.filter. robust, 26-28

ppa.filter. robust (robustness), 37

ppa.filter. robust, list-method (robustness), 37

ppa.in.silico, 29

ppa. iterate, $26-28,31,36,38,39$

ppa.iterate, list-method (ppa.iterate), 31

ppa. normalize, 26, 27, 31, 33, 35, 36, 38

ppa.normalize, list-method

(ppa. normalize), 33

ppa. unique, 26, 27, 35, 38

ppa. unique, list, list-method (ppa.unique), 35

robustness, 8,37

robustness, list-method (robustness), 37

scale, 3

sweep.graph (isa. sweep), 18

sweep.graph, list-method (isa.sweep), 18 